\title{
AIAA
}

\section{EFFECT OF DIRECTIONAL ARRAY SIZE ON THE MEASUREMENT OF AIRFRAME NOISE COMPONENTS}

\author{
Thomas F. Brooks and William M. Humphreys, Jr. \\ NASA Langley Research Center \\ Hampton, Virginia
}

AIAA Paper No. 99-1958

Presented at the

Fifth AIAA/CEAS Aeroacoustics Conference

May 10-12, 1999

Bellevue, Washington 
AIAA-99-1958

\title{
Effect of Directional Array Size On The Measurement of Airframe Noise Components
}

\author{
Thomas F. Brooks* \\ William M. Humphreys, Jr. ${ }^{\dagger}$ \\ NASA Langley Research Center \\ Hampton, Virginia 23681-0001
}

\begin{abstract}
A study was conducted to examine the effects of overall size of directional (or phased) arrays on the measurement of aeroacoustic components. An airframe model was mounted in the potential core of an open-jet windtunnel, with the directional arrays located outside the flow in an anechoic environment. Two array systems were used; one with a solid measurement angle that encompasses $31.6^{\circ}$ of source directivity and a smaller one that encompasses $7.2^{\circ}$. The arrays, and sub-arrays of various sizes, measured noise from a calibrator source and flap edge model setups. In these cases, noise was emitted from relatively small, but finite size source regions, with intense levels compared to other sources. Although the larger arrays revealed much more source region detail, the measured source levels were substantially reduced due to finer resolution compared to that of the smaller arrays. To better understand the measurements quantitatively, an analytical model was used to define the basic relationships between array to source region sizes and measured output level. Also, the effect of noise scattering by shear layer turbulence was examined using the present data and those of previous studies. Taken together, the two effects were sufficient to explain spectral level differences between arrays of different sizes. An important result of this study is that total (integrated) noise source levels are retrievable and the levels are independent of the array size as long as certain experimental and processing criteria are met. The criteria for both open and closed tunnels are discussed. The success of special
\end{abstract}

\footnotetext{
* Senior Research Scientist, Aeroacoustics Branch, Associate Fellow AIAA.

${ }^{\dagger}$ Research Scientist, Advanced Measurement and Diagnostics Branch, Senior Member AIAA.

Copyright (C) 1999 by the American Institute of Aeronautics and Astronautics, Inc. No copyright is asserted in the United States under Title 17, U.S. Code. The U.S. Government has a royalty-free license to exercise all rights under the copyright claimed herein for government purposes. All other rights are reserved by the copyright owner.
}

purpose diagonal-removal processing in obtaining integrated results is apparently dependent in part on source distribution. Also discussed is the fact that extended sources are subject to substantial measurement error, especially for large arrays.

\section{$\underline{\text { SYMBOLS }}$}

$A_{m} \quad$ shear layer refraction amplitude correction for $\mathrm{m}^{\text {th }}$ microphone

$c_{o} \quad$ speed of sound, $\mathrm{ft} / \mathrm{sec}$

$C_{l, n} \quad$ normalizing factor in integration equation, Eq.(5)

$\mathrm{dB} \quad$ sound pressure level, (ref. to $2 \times 10^{-5} \mathrm{~Pa}$ )

$\Delta \mathrm{dB}_{\mathrm{L}} \quad$ reduction in level due to array/source size effect

$\Delta \mathrm{dB}_{\mathrm{S}}$ reduction in level due to turbulence scattering

$\hat{e} \quad$ steering matrix, see Eq. (2)

$f \quad$ frequency, cycles/sec

$\hat{G} \quad$ cross-spectral matrix

$G_{i j} \quad$ cross spectra between $\mathrm{i}^{\text {th }}$ and $\mathrm{j}^{\text {th }}$ microphones, see Eq. (1)

$k \quad$ wavenumber $\left(=\omega / c_{o}\right), \mathrm{ft}^{-1}$

$l \quad$ integer

$L \quad$ analytical model source length, $\mathrm{ft}$

$m_{0} \quad$ total number of microphones in array

$M \quad$ tunnel free-jet Mach no. (=tunnel velocity $/ c_{o}$ )

integer

number of blocks of data

pressure, Pascals

array output power, mean square pressure, $\left(\overline{p^{2}}\right)$

$Q_{l^{\prime}, n^{\prime}} \quad$ sum of unit influences over integration region, see Eq. (5)

$r \quad$ radial distance, $\mathrm{ft}$ 


\begin{tabular}{|c|c|}
\hline$r_{c}$ & distance to center of array, ft, see Eq. (2) \\
\hline$R$ & distance from source to array surface, $\mathrm{ft}$ \\
\hline$t$ & time, sec \\
\hline$w$ & microphone weighting \\
\hline$\hat{W}$ & array weighting or shading matrix \\
\hline$W_{S}$ & spectral window weighting constant \\
\hline$X_{i k}, X_{j k}$ & $\begin{array}{l}\mathrm{k}^{\text {th }} \text { FFT data block for } \mathrm{i}^{\text {th }} \text { and } \mathrm{j}^{\text {th }} \\
\text { microphones }\end{array}$ \\
\hline$\vec{x}$ & location, $\mathrm{ft}$ \\
\hline$x, y$ & coordinates of scanning plane, $\mathrm{ft}$ \\
\hline$\delta$ & free-jet shear layer thickness, $\mathrm{ft}$ \\
\hline$\phi$ & elevation (or streamwise) angle, deg \\
\hline$\lambda$ & acoustic wavelength, $\mathrm{ft}$ \\
\hline$\theta_{D A}$ & $\begin{array}{l}\text { array size in terms of solid collection angle } \\
\text { with respect to the source position, deg }\end{array}$ \\
\hline$\theta_{0}$ & $\begin{array}{l}\text { offset angle of array with respect to the } \\
\text { center of the analytical line source, deg }\end{array}$ \\
\hline$\omega$ & frequency, $\mathrm{rad} / \mathrm{sec}$ \\
\hline$\omega \Delta t$ & $\begin{array}{l}\text { shear layer phase correction for } \omega \text {, radians, } \\
\text { see Eq. (2) }\end{array}$ \\
\hline$\psi$ & azimuthal (or sideline) angle, deg \\
\hline
\end{tabular}

Subscripts and Superscripts:

L lower limit

$T$ subscript denotes complex transpose of matrix

U upper limit

\section{$\underline{\text { INTRODUCTION }}$}

Single microphone measurements of aeroacoustic sources can be naturally hindered by poor signal-tonoise and by the inability to distinguish contributions from different source locations. This is especially true for model tests of airframe noise because the sources are non-propulsive and their magnitudes are similar in intensity level to test setup and tunnel noise sources. In the case of single airfoil-elements, it was found possible to localize and quantify trailing edge noise using distributions of individual microphones-but with processing techniques involving cross-spectral and coherentpower-output methods 1,2 . However, these techniques become cumbersome when studying multiple element sources. Starting in the same time frame (in the late 1970's) "acoustic mirror" systems $3,4,5$ were able to localize and, in number of cases, quantify the noise produced. For elliptic mirrors, a microphone is fixed at one elliptic focal point and the other focal point is placed in the source region of interest. However, determining source distributions about models requires mechanical movement of these sometimes very large systems. These systems are still found to be useful even today, especially in some larger test facilities 6,7 . Also in the 1970's, measurements involving directional (or phased) arrays of microphones were examined 8,9 using time delay and sum techniques. By adjusting for propagation time delays from particular source locations to the microphones, one is able to localize noise production in basically an equivalent fashion to that of the acoustic mirror approach, without the requirement to mechanically move the system. In the 1980's and early 1990's, an array technique using a frequency domain processing approach was introduced ${ }^{10,11}$ for a rotor noise application. Additional array applications for aeroacoustic measurements were made using time domain $^{12}$ and frequency domain ${ }^{13,14}$ approaches. In the mid 1990's, the use of arrays expanded. Using a ground array in a field study, flyover noise measurements of airframe noise have been made on landing aircraft in spite of the presence of engine noise ${ }^{15}$. For windtunnels, sophisticated array acquisition and processing systems were built for Boeing and NASA for closed $^{16}$ and open ${ }^{17}$ tunnel facilities. Efforts have been made to optimize array design and processing $18,19,20$, particularly to suppress array sidelobe interference in order to increase signal-to-noise and to reduce ambiguity in array results. Processing presently uses classical beamforming approaches in the frequency domain using cross-spectral matrices and robust steering algorithm codes $16,17,21$. Recent array applications have been conducted in the NASA Ames $40 \times 80 \mathrm{ft}^{7}$ and $7 \times 10 \mathrm{ft}^{22}$ tunnels, as well as in Boeing-Seattle's LSAF ${ }^{23}$ tunnel.

The studies, sited above, address array development and methodology as well as the measured results of aeroacoustic testing. However, the array literature fails to address how one obtains correct source amplitude. Mosher ${ }^{24}$ pointed this out in an extensive review of methodology. In aeroacoustics, the absolute level is important. A "perfect" array system would be able to determine correct location and amplitude of all sources under all conditions. Of course, such an array is impossible. For example, a proposal that arrays, given enough microphones and expanse, can reconstruct the noise source distribution within a region of space by surveying grid points electronically within the region is simply incorrect. Based on the Kirchhoff integral equation ${ }^{25}$ from fundamental acoustics theory, one cannot uniquely define interior noise sources knowing only what occurs at the boundary of the region. Then, certainly, neither could an array that encompasses only a small portion of such a boundary. In aeroacoustics, source distributions can 
be combinations of monopole, dipole, and quadrupole type sources. In order to construct a source distribution, one must hypothesize source types and geometry. Normally this is taken as a distribution of simple monopoles-with the additional provision that the monopoles be broadband-random and mutually independent. The array data is then processed using such assumptions. The extent to which such assumptions are true, or can be modeled as being true for a particular noise source, should determine whether the array gives a true measure of the phenomena under study.

Calibrations of arrays typically involve measuring noise from a small source (to approximate a point source) in an anechoic field. ${ }^{26}$ It is then established that the processed output of the array, when focussing or beamforming directly on the source, gives the same output as would a single microphone. Other focus points would give the output predicted from linear theory for the array geometry and source location. With this, it would appear that correct source levels could be measured if the sources are small, separate, incoherent, omni-directional and without high noise contamination. Others, such as distributed incoherent sources, have been taken (assumed) as being able to be measured correctly ${ }^{24}$. Even this basic proposition has not been validated. An illustration of uncertainty in amplitude measurement is contained in a study by Storms, et al. ${ }^{22}$ Spectra are presented from a large and a small array for a series of flap edge and slat noise phenomena. The levels measured by the small array were consistently higher (by some $6 \mathrm{~dB}$ at about $13 \mathrm{kHz}$ ). The spectra were obtained by volume integrations about the noise producing regions. The difference in levels were attributed to the difference in array size and a noise scatter effect from turbulence within the boundary layer of the wall, where the microphones are mounted.

The purpose of the present study is to help establish the effect of array size on the quantitative measurement of aeroacoustic sources. The arrays are mounted outside the flow in the anechoic free field. Noise directivity field variations over arrays of different sizes are determined. The measured noise sources are small but finite sized, with sufficient intensity compared to extraneous sources, so that the effects of relative size of the arrays and the source are clear. An analytical model of the array to source size effect is studied, as well as the effect of scatter due to shear layer turbulence. By using an integration approach over the noise regions, the degree to which each array can recover the energy of the source regions is determined. Implications of the results of the analysis are discussed.
In the following sections, the testing and processing approaches are defined and the processed data are then presented for three noise source configurations. Analyses are then given.

\section{AIRFRAME COMPONENT TEST AND PROCESSING}

\section{Test Setup and Method}

The tests were conducted in the Quiet Flow Facility (QFF) at Langley Research Center. The QFF is a quiet open-jet facility designed for anechoic acoustic testing. For the present airframe model testing, a 2 by 3-foot rectangular open-jet nozzle was used. The 3-foot span model was mounted between two side plates that were attached to sides of the nozzle exit. In Fig. 1, the model is visible through the Plexiglas windows located on the side-plates. The high-lift wing model is an instrumented NACA $63_{2}-215$ main element airfoil with a 30 percent chord half-span Fowler flap. This is approximately a 6 percent of a full-scale configuration, with a main element chordlength of 16 inches and a flap chordlength of 4.5 inches. For the data presented, the main element was aligned at $16^{\circ}$ angle of attack to the undisturbed flow and the flap was at $39^{\circ}$ relative to the main element. The noise source configurations studied

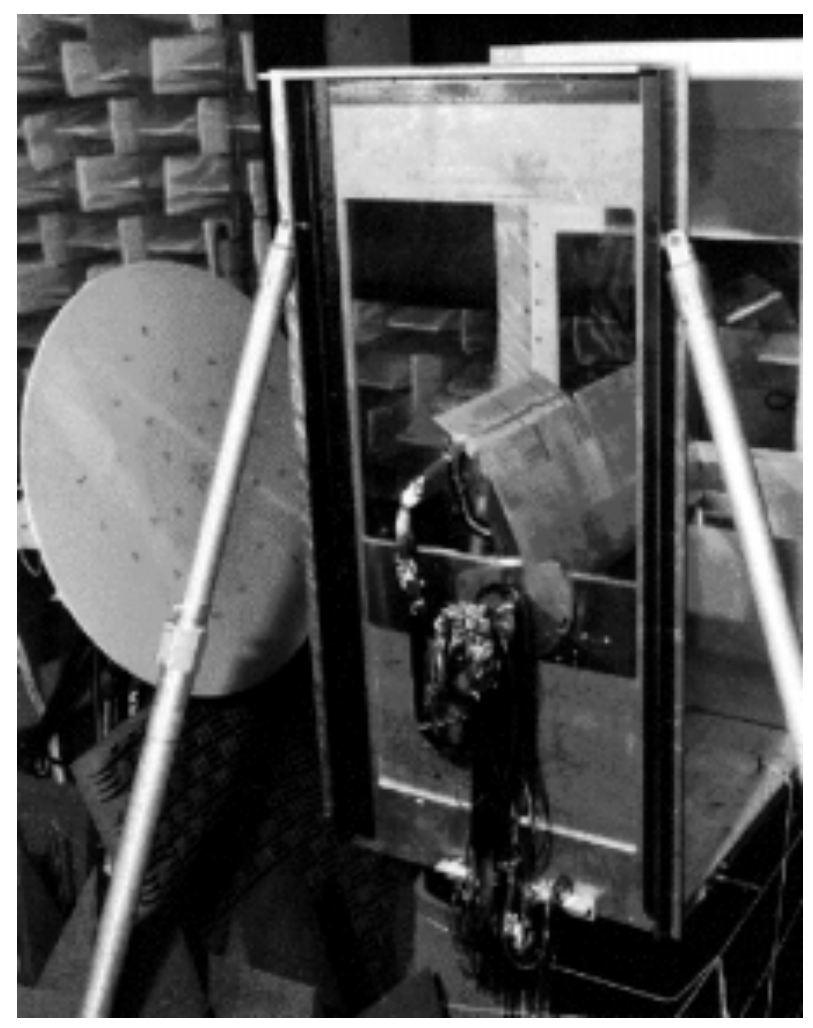

Figure 1. LADA mounted in the QFF on pressure side of model. 
are flap edges, with flat and contoured geometries, and a calibrator source placed next to the flap edge. Additional model, facility, and array details can be found in Refs. 27 and 17.

The Large Aperture Directional Array (LADA) was developed to identify dominant noise sources by producing high spatial resolution noise source localization maps along the airfoil surface. In Fig. 1, the LADA is shown mounted on the pressure side of the model-positioned 5 feet from the mid-span of the airfoil main-element trailing edge. It is constructed of a 4-foot diameter fiberglass panel to provide a flat surface to flush mount all microphones. The LADA incorporates 35 B\&K model 4135 1/4-inch microphones, spaced logarithmically in spiral patterns. The pattern design was based on one by Boeing ${ }^{20}$. It has five spirals of seven microphones each with the innermost microphones lying on a 1-inch radius and the outermost on a 17 -inch radius. With this radius, the array encompasses $31.6^{\circ}$ of solid collection angle. The solid angle, designated as $\theta_{D A}$, is a key parameter of this study. Sub-array groupings of the microphones of different radii are used in the analysis of the present study. The sub-array sizes are $\theta_{D A}=2.0^{\circ}, 9.9^{\circ}, 16.9^{\circ}$, $25.5^{\circ}$, and $31.6^{\circ}$, which are defined by LADA's inner 5 , $10,15,25$, and 35 microphones, respectively.

The Small Aperture Directional Array (SADA) is used to measure the acoustic directivity and spectra of selected portions of the wing-flap model. SADA is shown in Fig. 2 mounted on a pivotal boom on the suction side of the model. The pivotal boom is moved to position SADA about the model for the directivity measurements. The aperture of the array is small in order that all microphones in the array remain within approximately the same model noise directivity at any elevation or azimuth position. The SADA consists of

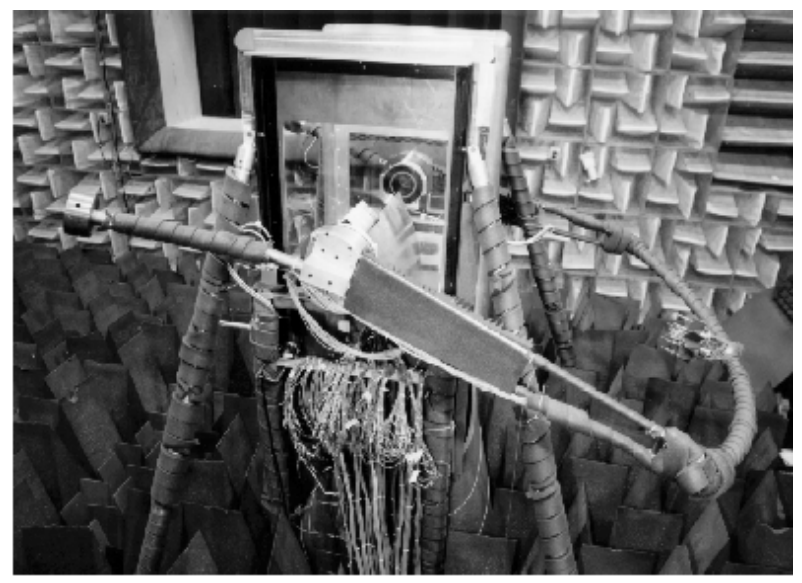

Figure 2. SADA mounted on pivotal boom in the QFF on suction side of model.
33 B\&K model 4133, 1/8-inch microphones projecting from an acoustically treated aluminum frame. The array pattern incorporates four irregular circles of eight microphones each and one microphone at the center of the array. Each circle is twice the diameter of the circle it encloses. The maximum radius of the array is 3.89 inches. With the SADA positioned 5 foot from the model, solid collection angles of $\theta_{D A}=1.8^{\circ}, 3.6^{\circ}$, and $7.2^{\circ}$ are defined by the inner, middle, and outer subarrays. Because of the need (for the directivity measurements) to keep the array resolution constant and independent of frequency, special blended processing is used for the SADA. This effectively makes $\theta_{D A}$ a function of frequency (inversely proportional to frequency).

In Fig. 3, the SADA measurement positions for zero azimuthal angle $\left(\psi=0^{\circ}\right)$ are drawn in a side view (opposite side to that of Fig. 2) of the test setup. The position of SADA in the photo of Fig. 2 corresponds to elevation angle $\phi=-124^{\circ}$ in the drawing. For this paper, measurements for the pressure side are presented. In Fig. 3, the SADA is seen positioned at $\phi=107^{\circ}$ and an inset of the microphone-coverage region of the LADA is shown superimposed at its own $\phi=106^{\circ}$ position. In practice, the two systems were never operated together. The open jet shear layer boundaries (defined at 10 and $90 \%$ of the potential core velocity) are shown as measured along the $\psi=0^{\circ}$ plane. A mean shear line or surface is shown, which is part of a curved three-dimensional mean shear surface defined mathematically from the shear layer

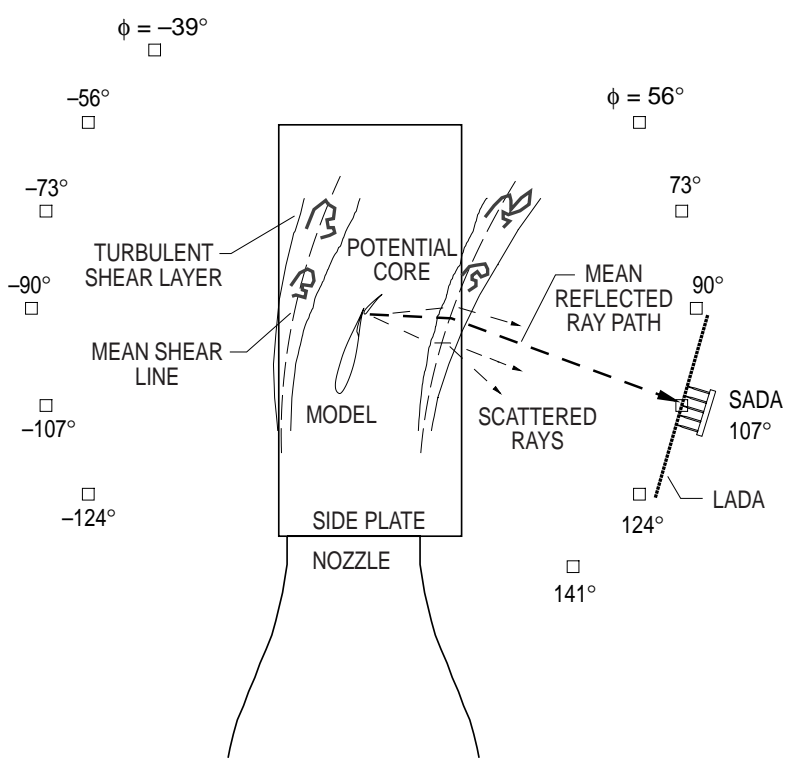

Figure 3. Scale drawing of test setup and shear layer. Noise ray paths from the source to the microphones are illustrated. 
measurements. This is mentioned with regard to the beamformer solution to follow. Also illustrated, in Fig. 3 , are scattered noise ray paths, which are dealt with in the analysis section.

\section{Data Acquisition and Post-Processing}

As described in Ref. 17, both arrays employed acquisition hardware consisting of transient data recorders controlled by a workstation. All data channels were simultaneously recorded with a 14-bit dynamic range at a sampling rate of $142.857 \mathrm{kHz}$. Two million 2-byte samples were taken for each acquisition. The signals from each microphone channel were conditioned with high pass filters set to $300 \mathrm{~Hz}$ and with antialiasing filters set at $50 \mathrm{kHz}$, which is substantially below the $71.43 \mathrm{kHz}$ Nyquist frequency.

Microphone calibration data were accounted for in post-processing. For the SADA, regular pistonphone and injection calibrations were made for all the individual microphones. The manufacturer specifications for frequency responses, based on mounting technique, were used for both the SADA and LADA. For the LADA, because of the difficulty of performing regular individual pistonphone calibrations and accounting for flush-mounting details of the microphones, a calibration procedure somewhat similar to that of Ref. 26 was used. Here, the in situ responses to reference sources were compared to isolated free-field measurements. The phase response, for all individual microphones, was adjusted by small time delay offsets in the beamform processing. For the amplitude response, a single amplitude calibration adjustment was used, for all microphones and frequencies, based on comparison of matched single and multiple microphone groups of the acoustically treated SADA.

Post processing of the data begins with the computation of the cross-spectral matrix for each data set. The computation of the individual matrix elements is performed using Fast Fourier Transforms (FFT) of the original data ensemble. This is done after converting the raw data to engineering units. The time data is segmented into a series of non-overlapping blocks (244 blocks for the present data) each containing 8192 samples. Using a Hamming window, each of these blocks of data is Fourier transformed into the frequency domain with a frequency resolution of $17.45 \mathrm{~Hz}$. The individual cross spectrum for microphones $i$ and $j$ are

$$
G_{i j}(f)=\frac{1}{N W_{s}} \sum_{k=1}^{N}\left[X_{i k}^{*}(f) X_{j k}(f)\right]
$$

where $W_{s}$ is the data-window weighting constant, $N$ is the number of blocks of data, and $X$ represents an FFT data block. The full matrix is, with $m_{0}$ being the number of microphones

$$
\hat{G}=\left[\begin{array}{cccc}
G_{11} & G_{12} & \cdots & G_{1 m_{0}} \\
& G_{22} & & \vdots \\
& & \ddots & \vdots \\
& & & G_{m_{0} m_{0}}
\end{array}\right]
$$

The lower triangular elements of this Hermitian matrix are determined from taking the complex conjugates of the upper triangular elements.

\section{Beamforming}

A conventional beamforming approach is used to electronically "steer" the array to chosen noise source locations. For each selected steering location, a steering vector containing an entry for each microphone in the array is defined as

$$
\hat{e}=\left[\begin{array}{c}
A_{1} \frac{r_{1}}{r_{c}} \exp \left\{j\left[\left(\vec{k} \cdot \vec{x}_{1}\right)+\omega \Delta t_{1, \text { shear }}\right]\right\} \\
\vdots \\
A_{m_{0}} \frac{r_{m_{0}}}{r_{c}} \exp \left\{j\left[\left(\vec{k} \cdot \vec{x}_{m_{0}}\right)+\omega \Delta t_{m_{0}, \text { shear }}\right]\right\}
\end{array}\right]
$$

where $\vec{k}$ is the acoustic wave vector, $\vec{x}_{m}$ is the distance vector from the steering location to each microphone $\mathrm{m}$, and $\omega$ is the frequency, in radians/sec, $(=2 \pi f)$. Equation (2) contains terms to account for mean amplitude and phase changes due to refracted sound transmission through the shear layer to the individual microphones of the arrays. A mean refracted ray path is illustrated in Fig. 3. The correction terms are calculated $^{17}$ by the use of Snell's law in Amiet's method $^{28,29}$, adapted to a curved three-dimensional mean shear surface defined in the shear layer. In Eq. (2), the ratio $\left(r_{m} / r_{c}\right)$ is included to normalize the distance related amplitude to that of the center microphone at $r_{c} . \quad A_{m}$ is the refraction amplitude correction. Correspondingly, $\omega \Delta t_{\text {m, shear }}$ is the phase correction for microphone $m$. ( $\Delta t_{m \text {,shear }}$ is the additional time (compared to a direct path) it takes an acoustic ray to travel to a microphone from the steering location, due to the convection by the open jet flow and refraction by the shear layer.) Equation (2) corrects details of the corresponding equation in Ref. 17. 
The output power spectrum (or response) of the array at the steering location is obtained from

$$
P(\hat{e})=\frac{\hat{e}^{T} \hat{G} \hat{e}}{m_{0}^{2}}
$$

where the subscript $T$ denotes a complex transpose of the matrix. Here $P(\hat{e})$ is a mean-squared-pressure quantity. Note that the cross-spectral matrix normally has a corresponding background matrix subtracted from it to improve fidelity ${ }^{17}$. The division by the number of microphones-squared serves to reference levels to an equivalent single microphone measurement. $P(\hat{e})$ is determined for each narrowband frequency (here at 17.45 $\mathrm{Hz}$ resolution bandwidth) of interest. Wider bands are obtained by summing power, after the operations of Eq. (3) are performed.

For the SADA, a special shading algorithm is normally used when directivity and spectral measurements are made. This keeps the array beamwidth invariant, thereby providing a constant sensing area over noise source regions ${ }^{17,10}$. This prevents the need to correct measured levels, because resolution (sensing area) does not change with frequency and it is large enough to enclose the source regions of interest. In this blending application, the inner microphone groups (or subarrays) are made inactive at low frequencies and the outer microphones are made inactive at high frequencies. The resultant shaded or blended steered response is

$$
P(\hat{e})=\frac{\hat{e}^{T} \hat{W} \hat{G} \hat{W}^{T} \hat{e}}{\left(\sum_{m=1}^{m_{0}} w_{m}\right)^{2}}
$$

where $w_{m}$ is the frequency dependent shading (or weighting) for each microphone $m . \hat{W}$ is a row matrix containing the $w_{m}$ terms. For the blended case of the SADA, the number of active microphones is always 17 , so the denominator is $(17)^{2}$. Note that for the present paper, the weighting terms are used for both the SADA and LADA to define sub-arrays of different sizes, although frequency invariant beamforming was not applied to the LADA. When all $w_{m}$ terms are set to one and $W$ becomes an identity matrix, all microphones are fully active in the beamforming.
A modified form of Eq. (4) is commonly used ${ }^{24}$ to improve dynamic range of the array results in bad signal-to-noise tunnel applications. The primary intent is to remove microphone self noise (or pseudo-noise) contamination. This involves removing the diagonal terms of $\hat{G}$ and accounting for the change in the number of terms of $\hat{G}$ in the denominator. For this case, the beamform patterns are modified from that of Eq. (4). This "diagonal-removal" method is, at least as defined in this paper.

$$
P(\hat{e})=\frac{\hat{e}^{T} \hat{W} \hat{G}_{\text {diag }=0} \hat{W}^{T} \hat{e}}{\left(\sum_{m=1}^{m_{0}} w_{m}\right)^{2}-\left(\sum_{m=1}^{m_{0}} w_{m}\right)}
$$

\section{Source Region Integration}

For this paper, the array response is determined, using Eq. (4), for a range (grid) of steering (or scanning) locations over a plane that is positioned through the airfoil main element. For particular frequencies, contours of the response levels are plotted over the plane. In the case of an ideal point source in the plane (in free space without reflections), the contour would have the appearance of the theoretical array beampattern projected onto the plane. The point source location would exhibit the maximum level, representing the total output of the source. In the case of distributed sources, the total output must result from an integration over a specified source region. However, in the integration the mutual summed influence of the distributed sources, each with its own array related beampattern, must be taken into account (or normalized out). This could be viewed as a way to avoid "double-counting" of source contributions. The following integration approach accounts for these influences in a systematic way by incorporating the beamformer algorithm.

We define the coordinates of grid points in a scanning plane as $(x, y)=\left(x_{0}+(l) \Delta x, y_{0}+(n) \Delta y\right)$, where $\Delta x$ and $\Delta y$ are grid spacing and $l$ and $n$ are integers. The integration region covers the area defined by $x_{0}+\left(l_{L}\right) \Delta x$ to $x_{0}\left(l_{U}\right) \Delta x$ and $y_{0}+\left(n_{L}\right) \Delta y$ to $y_{0}+\left(n_{U}\right) \Delta y$. The integration approach is readily accomplished over a volume but is introduced here over a plane for simplicity. At these grid points, let $P_{l, n}$ 
represent $P(\hat{e})$. Let $P_{T}$ be the integrated (meansquared-pressure) output of the region, which is

$$
\begin{aligned}
& P_{T}=\sum_{l=l_{L}}^{l_{U}} \sum_{n=n_{L}}^{n_{U}}\left[P_{l, n} / C_{l, n}\right] \\
& C_{l, n}=\left[\sum_{l^{\prime}=l_{L}^{\prime}}^{l_{U}^{\prime}} \sum_{n^{\prime}=n_{L}^{\prime}}^{n_{U}^{\prime}} Q_{l^{\prime}, n^{\prime}}\right]_{l, n} \\
& Q_{l^{\prime}, n^{\prime}}=\left[\frac{\hat{e} T \hat{W} \hat{G}_{l, n}^{\prime} \hat{W}^{T} \hat{e}}{\left(\sum_{m=1}^{m_{0}} w_{m}\right)^{2}}\right]_{l^{\prime}, n^{\prime}} \\
& \hat{G}_{l, n}^{\prime}=\left[\begin{array}{cccc}
\left(e_{1}^{*} e_{1}\right)^{-1} & \left(e_{1}^{*} e_{2}\right)^{-1} & \cdots & \left(e_{1}^{*} e_{m_{0}}\right)^{-1} \\
& \left(e_{2}^{*} e_{2}\right)^{-1} & & \vdots \\
& & \ddots & \vdots \\
& & & \left(e_{m_{0}}^{*} e_{m_{0}}\right)^{-1}
\end{array}\right]_{l, n}
\end{aligned}
$$

It is seen that $P_{T}$ is determined by summing the values $P_{l, n}$ after being normalized by corresponding $C_{l, n}$ values . $C_{l, n}$ accounts for the integrated beampattern characteristics of the array over the region with respect to the $l, n$ location. It is the sum of unit influences $Q_{l^{\prime}, n^{\prime}}$ from all other locations in the region. The use of inverses of the $l, n$-location steering vectors in the synthesized cross-spectral matrix $\hat{G}_{l, n}^{\prime}$ accounts for the beamform characteristics, including side lobe inclusion and shear layer correction. A roughly uniform distributed source strength is assumed over the integration region, although the sides of the main beampattern may extend beyond. One could simplify the above calculations by using a representative $C_{l_{0}, n_{0}}$ to replace the individual values of $C_{l, n}$. This is especially appropriate for compact sources and reduces computation time greatly. The use of $C_{l_{0}, n_{0}}$ (simplified method) should be equivalent to integration methods that have been employed by Mosher ${ }^{24}$ and Dougherty. Equation (5) is not exact, but should produce good results as long as the integration area contains no significant contributions, including portions of main lobes or side lobes, from sources outside the area. The procedure implicitly assumes that the source regions are comprised only of a distribution of statistically independent (uncorrelated) point noise sources, where spatially pressure-squared summing is appropriate. Equation (5) can be used for volume integration by simply expanding the summation to stacked multiple scanning planes.

An equivalent to Eq. (4a) can be obtained for Eq. (5). For this "diagonal-removal" method, $P_{T}$ is obtained by Eq. (5), except that $Q_{l^{\prime}, n^{\prime}}$ becomes

$$
Q_{l^{\prime}, n^{\prime}}=\left[\frac{\hat{e}^{T} \hat{W}\left(\hat{G}_{l, n}^{\prime}\right)_{\text {diag }=0} \hat{W}^{T} \hat{e}}{\left(\sum_{m=1}^{m_{0}} w_{m}\right)^{2}-\left(\sum_{m=1}^{m_{0}} w_{m}\right)}\right]_{l^{\prime}, n^{\prime}}
$$

In the analysis of results section, Eq. (5) and its related simplified method are used and evaluated.

\section{MEASURED RESULTS}

\section{SADA Free-Field Directivity}

For the SADA at the $\phi=107^{\circ}$ position, Fig. 4 shows a theoretical contour plot over the model of the spatial noise admittance (or negative rejection) in $\mathrm{dB}$ level. The array is steered to the intersection of the airfoil main element and the flap edge. The effective sensing area is defined as that region within the $-3 \mathrm{~dB}$ contour on the main beampattern lobe. The rejection of

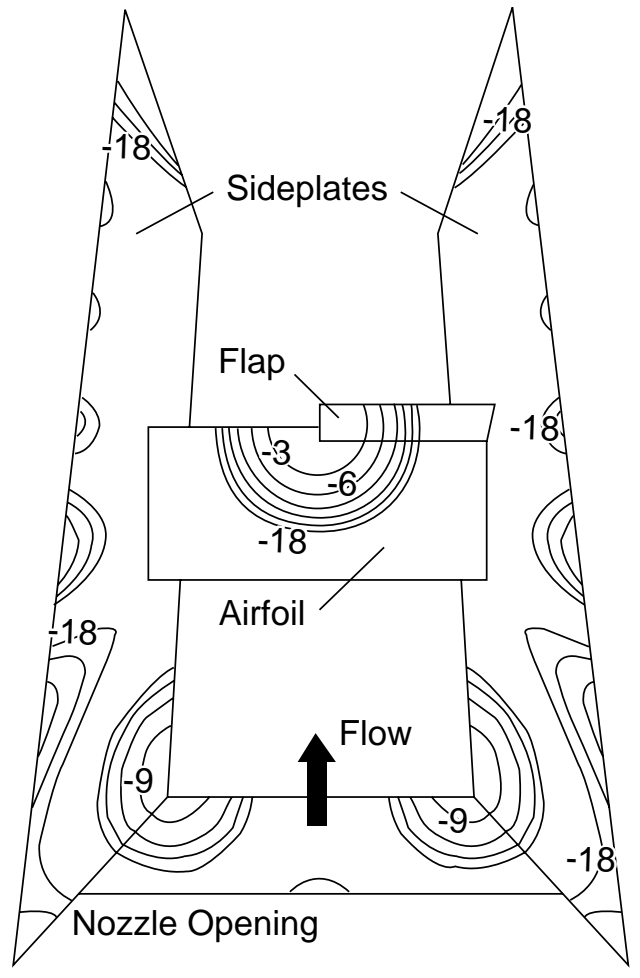

Figure 4. SADA admittance contour map over model pressure side. This is a "bird's eye" view of model test apparatus from the SADA. 
(extraneous) noise regions over the side plates and nozzle opening is also shown. The contour is for the frequencies 10, 20, and $40 \mathrm{kHz}$. For frequencies between these, the blended processing keeps the sensing area approximately the same as that shown ${ }^{27}$. At lower and higher frequencies than this range, the sensing area becomes wider and narrower, respectively. Therefore, over a broad range of frequencies, the spectral output of the SADA should represent only that noise which is radiated from the flap-edge region. Noise directivity is mapped by placing the SADA at a series of elevation and azimuthal angles.

Figure 5 shows the model with the flap-edge directivity contour mapped over a spherical surface, defined by the SADA positions. The measurements are for a flap, with a flat cutoff-shaped edge, placed at a $39^{\circ}$ angle to the main wing element. For the $6.3 \mathrm{kHz}$ onethird octave frequency band shown, the directivity on the pressure (flyover) side of the model is most intense "underneath" the model. On the suction side of the model, the levels are less but are seen to increase in the downstream direction. Figure 6 are pressure-side directivity maps for different frequencies. These maps are the flattened spherical surfaces shown in Fig. 5. The positive azimuthal angles $\psi$ are on the flap side of the model. The elevation angles $\phi$ with the smaller values (at the top of the plots) are in the downstream direction. For each set of three one-third-octave directivity maps, sketches of the respective calibrator source, flat edge,

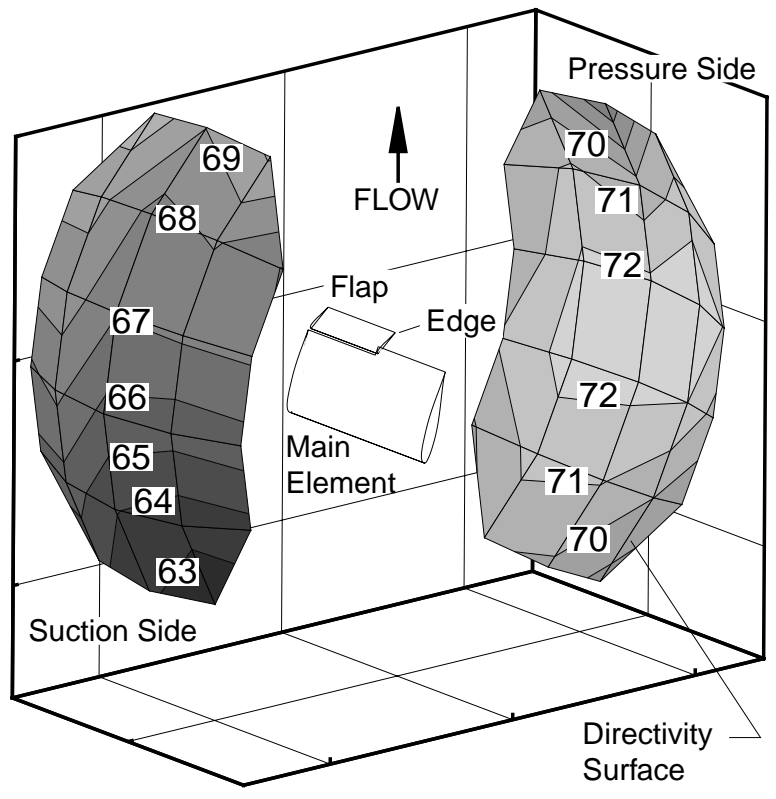

Figure 5. Directivity contour levels over "surfaces" defined by SADA measurements. One-third octave levels for $f_{1 / 3}=6.3 \mathrm{kHz}$. and contoured edge configurations are shown. The calibrator source is the open end of a one-inch diameter tube, which for low frequencies should approximate a simple monopole source. When the calibrator source is not present, the edges are the noise producing regions of the flap (this edge noise is of primary interest in the study of the airframe noise problem). In Fig. 6, the outlines of the SADA and the microphone-coverage region of the LADA are superimposed. This is intended to show the positions used for most of the data presented in this paper and the directivity variations present over the face of the arrays.

For the calibrator source, both the $M=0$ and 0.17 directivities show "hot spots" on the azimuthal side which is opposite the flap. This is an apparent reflection/shielding effect due to the source position being next to and slightly behind the flap edge. Except at the high frequencies, the basic directivity characteristics do not seem to be substantially affected by the flow. This tendency will be used in the analysis of turbulent shearlayer noise scatter subsequently. For the flat and contoured flap-edge configurations, the directivities are generally uniform at lower frequencies. At higher frequencies, however, stronger variations are seen. In the later sections, the spectral results from the LADA are to be compared to that of the SADA - to examine the effect of array size. These directivity results, as well as those of the point source, suggest that such comparisons are proper because the levels at the SADA appear to approximate some "average" of levels over the face of the LADA. This conclusion assumes that no significant phase variations occur over the face of the LADA. A limited review of phase data did not reveal any significant variations.

\section{$\underline{\text { Source Distribution and Spectra for Different Array }}$ $\underline{\text { Sizes }}$}

Figure 7 shows SADA and LADA source distribution contour maps for the model configurations at different tunnel speeds. The levels shown are for the 40 $\mathrm{kHz}$ one-third octave band. The LADA is using all 35 microphones, so $\theta_{D A}=31.6^{\circ}$, and at this frequency, $\theta_{D A}=1.8^{\circ}$, for the SADA. The contours were created by electronically steering (focussing) to predefined grid points, spaced $1 / 4$ inch apart, on a plane projected through the chordline of the main-element model.

For the calibrator source for $M=0$, the LADA gives a well-defined contour that clearly locates the source alongside the flap edge. The dynamic range over the spatial region (about $13 \mathrm{~dB}$ ) is good and side lobes are seen to be projected to within 4 inches of the main lobe at the source. The SADA contour is dominated by the 

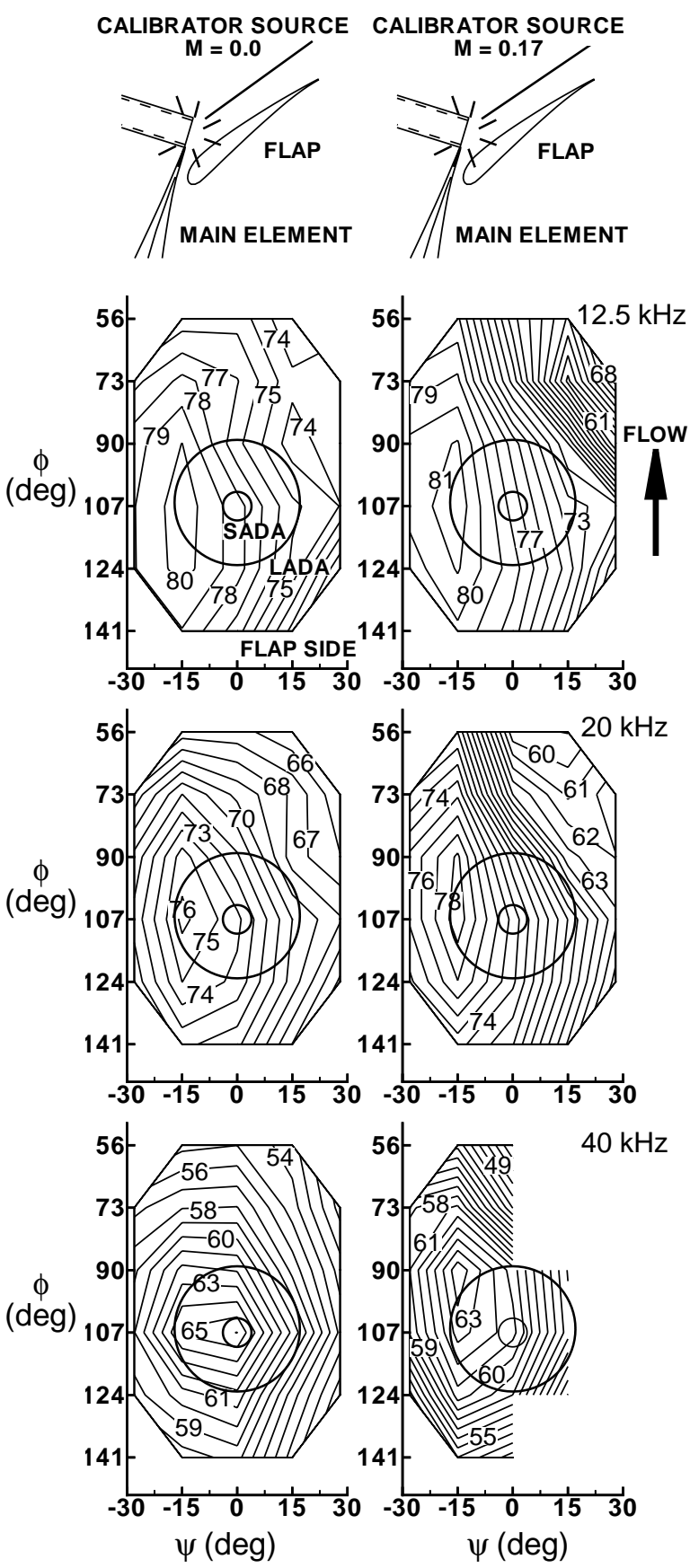

(a) calibrator source for $M=0$ and 0.17 .
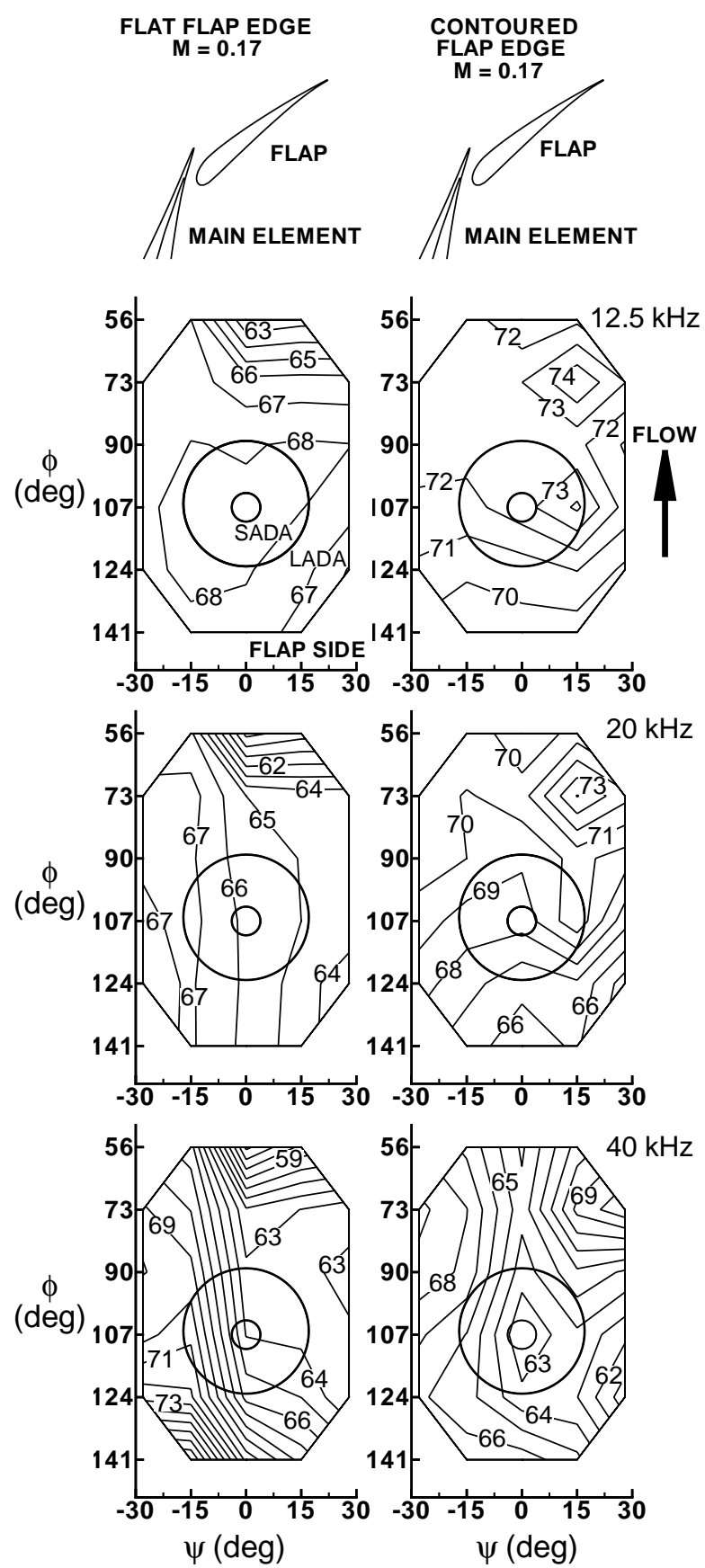

(b) flat edge flap and contoured edge flap for $M=0.17$.

Figure 6. Directivity levels for calibrator source and flat-edge and contoured-edge flaps for three one-third octave bands.

main lobe, which is properly centered at the source location. For non-zero Mach number, the LADA still properly locates the source by the use of the shear-layer refraction corrections in the steering vector processing. However, the maximum level drops and the dynamic range drops to about $7 \mathrm{~dB}$ for $M=0.11$ and $5 \mathrm{~dB}$ for $M=0.17$. The LADA maximum levels are reduced and the image is more dispersed with increased speed. The SADA levels are less reduced and any dispersion is less noticeable because of the broader beampattern. The contours for the flat and contoured flap edges suggest a concentrated source distribution of an inch or two for this particular frequency. Note that the dynamic range is poor at about $5 \mathrm{~dB}$ for $M=0.11$ and 2 to $3 \mathrm{~dB}$ for 


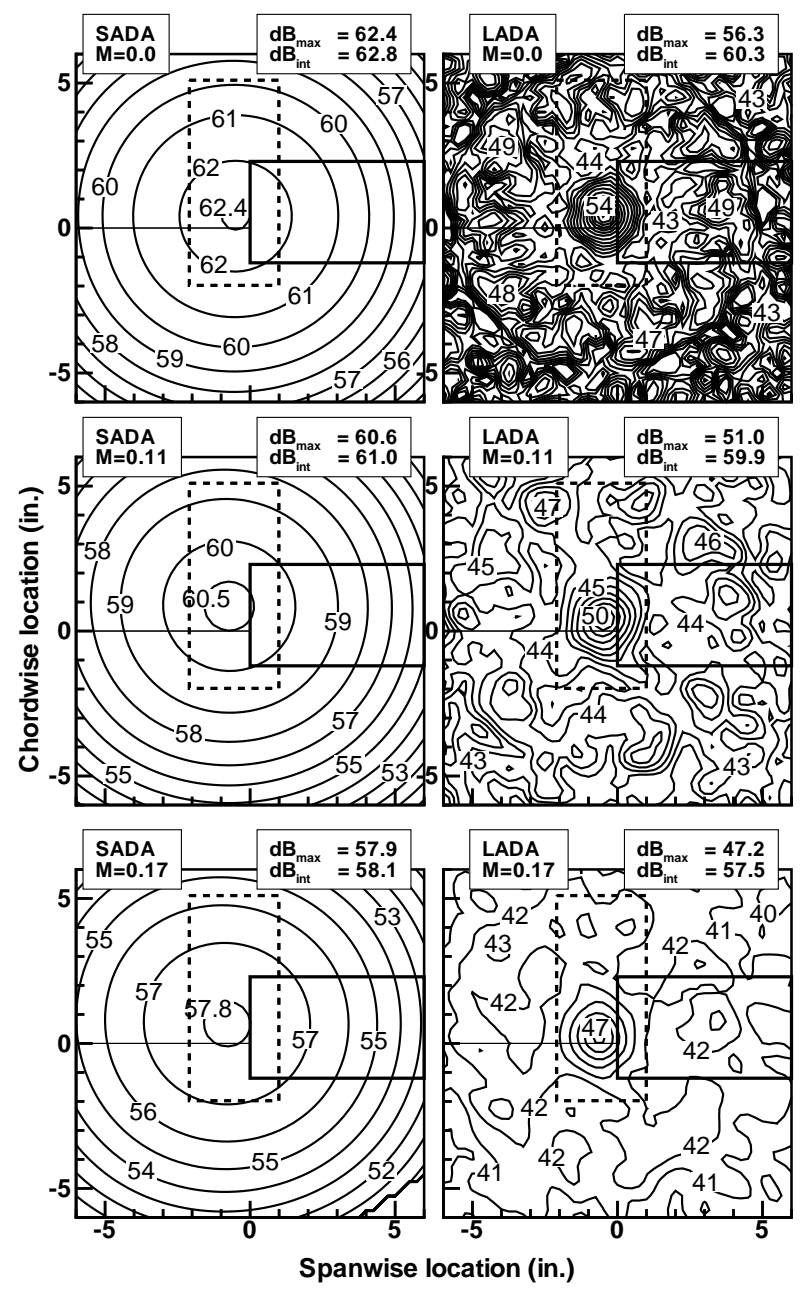

(a) calibrator source for $M=0,0.11$, and 0.17 .

Figure 7. Source distribution contours over the flapedge region, using the SADA and LADA. $f_{1 / 3}=40 \mathrm{kHz}$. Integration areas are shown.

$M=0.17$. The SADA does not have the resolution to define the source distribution details. Note, as with the calibrator source, the LADA maximum levels are lower than that of the SADA. In Fig. 7, integration areas are defined by the dashed-line boxes. Integration procedures are discussed in the analysis of results section.

Note that the dynamic range shown for the LADA in Fig. 7 can be improved by the "diagonal-removal" method of Eq. (4a). These results are not shown. But when such processing is done, the peak values $\mathrm{dB}_{\max }$ are lowered in amplitude by (starting with the lower Mach numbers): $-0.6,-0.1$, and -0.2 for part (a); -0.3 and -0.2 for (b); and -1.9 and -4.2 for (c). These drops in level appear to depend somewhat on the source

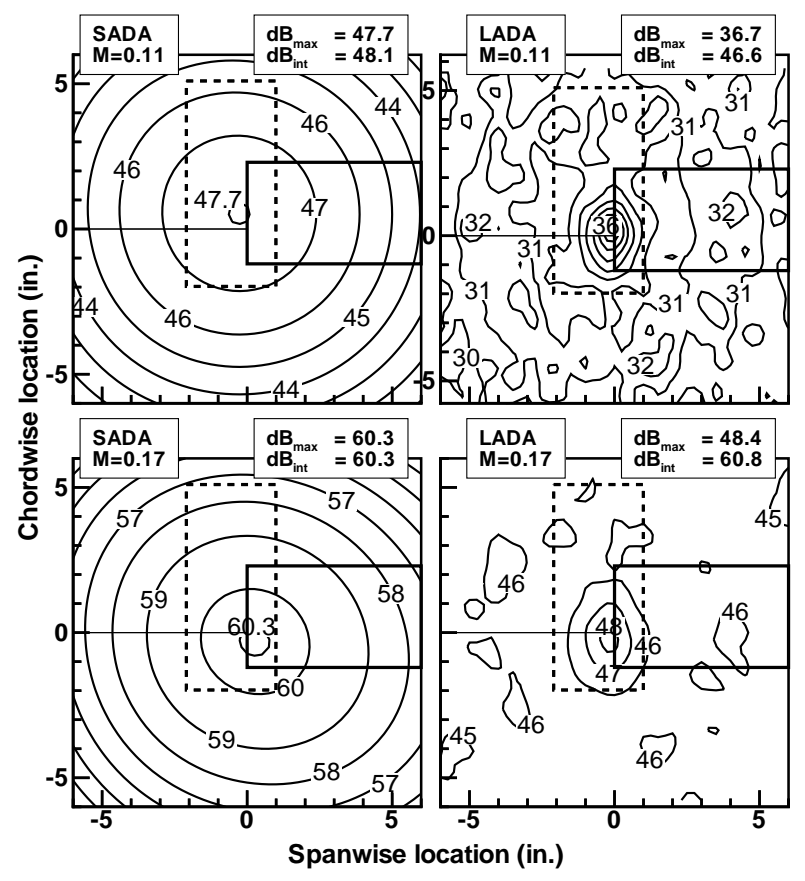

(b) flat-edge flap for $M=0.11$ and 0.17 .

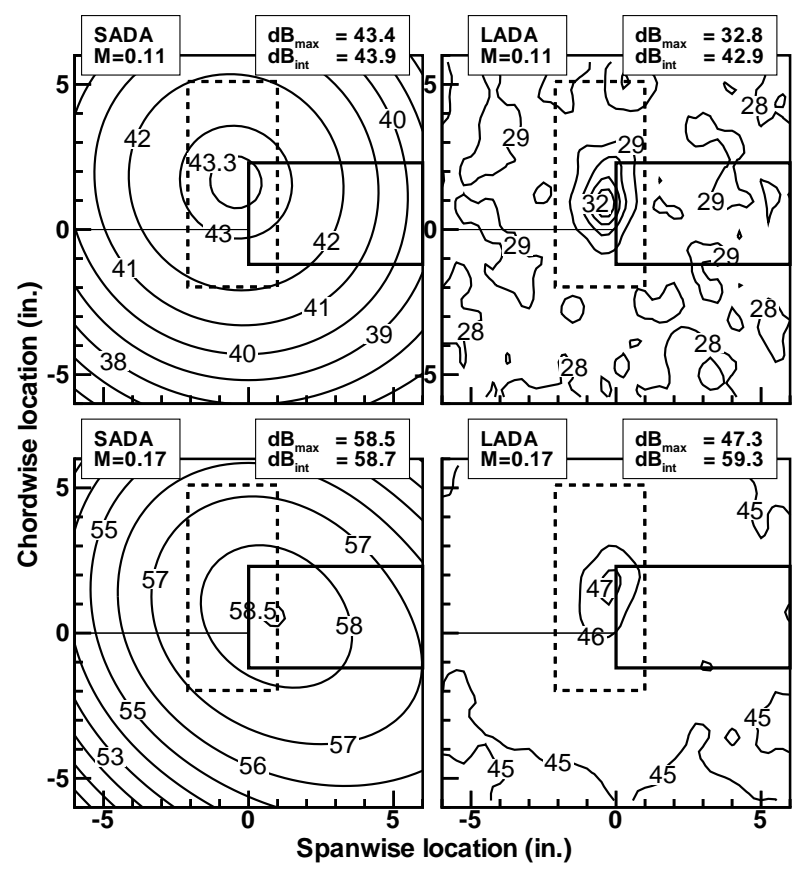

(c) contoured-edge flap for $M=0.11$ and 0.17 .

Figure 7. Concluded.

distribution. The noise floor level in the contour is greatly dropped to include negative $P(\hat{e})$ values between the main and side lobes of the beampattern. It is beyond the scope of this study to fully evaluate the 
diagonal-removal method, but integration results are presented in the next section for comparison to that of standard processing.

The amplitude effect of array size is demonstrated in Fig. 8 for the flat-edge flap at $M=0.17$. The standard processing of Eq. (5) is used. Spectra are presented for the SADA and LADA using different sub-arrays of microphones to define different $\theta_{D A}$. Each spectral level corresponds to the maximum (peak) levels near the respective contours, such as those of Fig.7. The spectra are comprised of only 7 one-third octave bands to simplify processing and presentation. The sub-array sizes $\theta_{D A}=2.0^{\circ}, 9.9^{\circ}, 16.9^{\circ}, 25.5^{\circ}$, and $31.6^{\circ}$, are defined by the LADA's inner microphones, as previously described. For the SADA, $\theta_{D A}=1.8^{\circ}, 3.6^{\circ}$, and $7.2^{\circ}$ are defined by the inner, middle, and outer sub-arrays, respectively. Figure 8 demonstrates a key characteristic of array size-that reductions in level occur with increases in frequency and array solid measurement angle $\theta_{D A}$. The results are very consistent, although some differences are seen between the SADA and the LADA at similar (small) $\theta_{D A}$ values, particularly at high frequencies. This may be due in part to slight model differences between the tests. It is noted that in the 40 and $50 \mathrm{kHz}$ one-third octave bands that such specifics as flap surface smoothness can affect the noise (see Ref. 27). Other factors likely include unforeseen, and thus not fully accounted for, high frequency response differences of the microphones and the details of the different mountings. In any case, the basic trend of the data of Fig. 8 is quite clear.

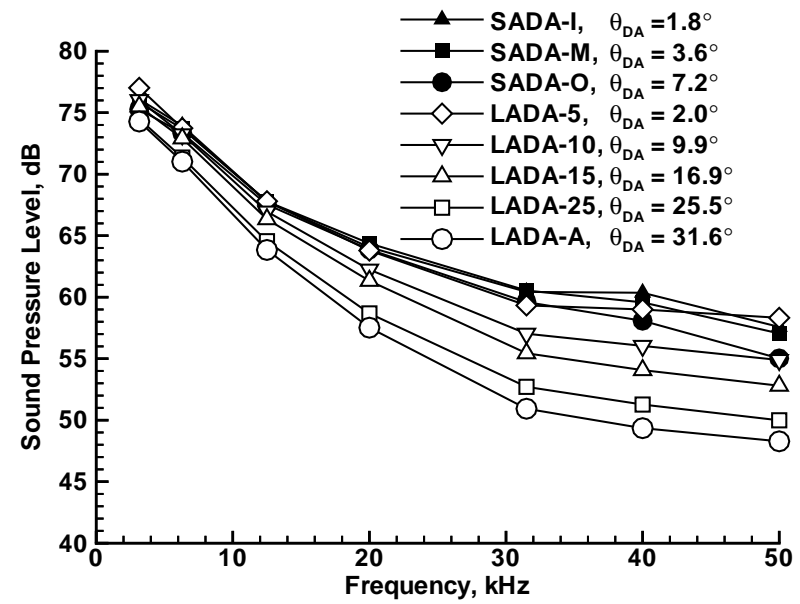

Figure 8. One-third octave spectra determined using the arrays and sub-arrays of different size. Case shown is the flat edge flap at $M=.17$.

In a presentation similar in order to Fig. 7, Fig. 9 shows spectral comparisons for single microphones at center locations of the arrays, as well as the LADA using all 35 microphones and the SADA using the blended processing. (These are the spectra represented by lines.) For the calibrator source at all speeds, the signal was generally well above the background noise, except at the higher frequencies. The single microphones, for both systems, and the SADA give similar spectra. This demonstrates that the SADA, with blended processing, functions properly as an equivalent single microphone, for that portion of noise radiated from the focussed source region. However, as could be anticipated from the results of Fig. 8, the LADA measurements are substantially lower at high frequencies, more so when the tunnel speed is increased. For the two flap edge noise cases, one sees that the SADA results are generally 3 to $5 \mathrm{~dB}$ lower than the single microphone results. This shows that the flap-edge noise (which is measured without the need for correction by the SADA) is below the total noise levelswhich simply demonstrates the general need for such array measurements that allows one to extract flap-edge noise from the difficult environment of the total noise field. Also shown, for the flap edge cases, are the lower levels for spectra that is obtained from the LADA. In the following section, the reasons for the lower levels and the use of integration methods to account for the levels are examined.

\section{$\underline{\text { ANALYSIS }}$}

\section{$\underline{\text { Some Factors Affecting Measurements }}$}

Before the measured results are analyzed, several basic factors that affect measurement and its interpretation are examined.

Effect of array size on the measurement of ideal line source: An analytical model is used to examine the effect of array size and orientation on the measurement of noise from an ideal line source. The line source considered is a distribution of mutually-uncorrelated monopoles of uniform strength over a length $L$. Figure 10 shows a sketch of a line source that is positioned at the center of a spherical surface, of radius $R(L<<R)$, over which a circular "array" is defined. The array encompasses a solid angle $\theta_{D A}$ of the line source directivity with respect to a normal to the line. It is offset by an angle $\theta_{0}$. "Array measurements" are made by integrating the theoretical cross spectra of the noise over the surface in a manner consistent with experimental data processing. The integration makes the effective number of "microphones" equal to infinity. Such differences as number of microphones in this reference array and the actual arrays have little impact on the general conclusions that will be drawn from this 
SADA Single Microphone at Center

SADA Blended Array

LADA Single Microphone at Center

LADA Full Array

Integrated LADA Spectra

Integrated SADA Spectra

Integrated LADA Spectra Using $\mathrm{C}_{\mathrm{o}}, \mathrm{n}_{\mathrm{O}}$

Integrated SADA Spectra Using $\mathrm{C}_{\mathrm{I}_{0}, \mathrm{n}_{0}}$

Integrated LADA Spectra Using $\mathrm{C}_{\mathrm{l}_{0}, \mathrm{n}_{0}}$, No Diagonal

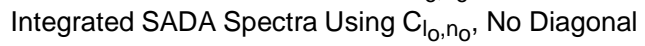
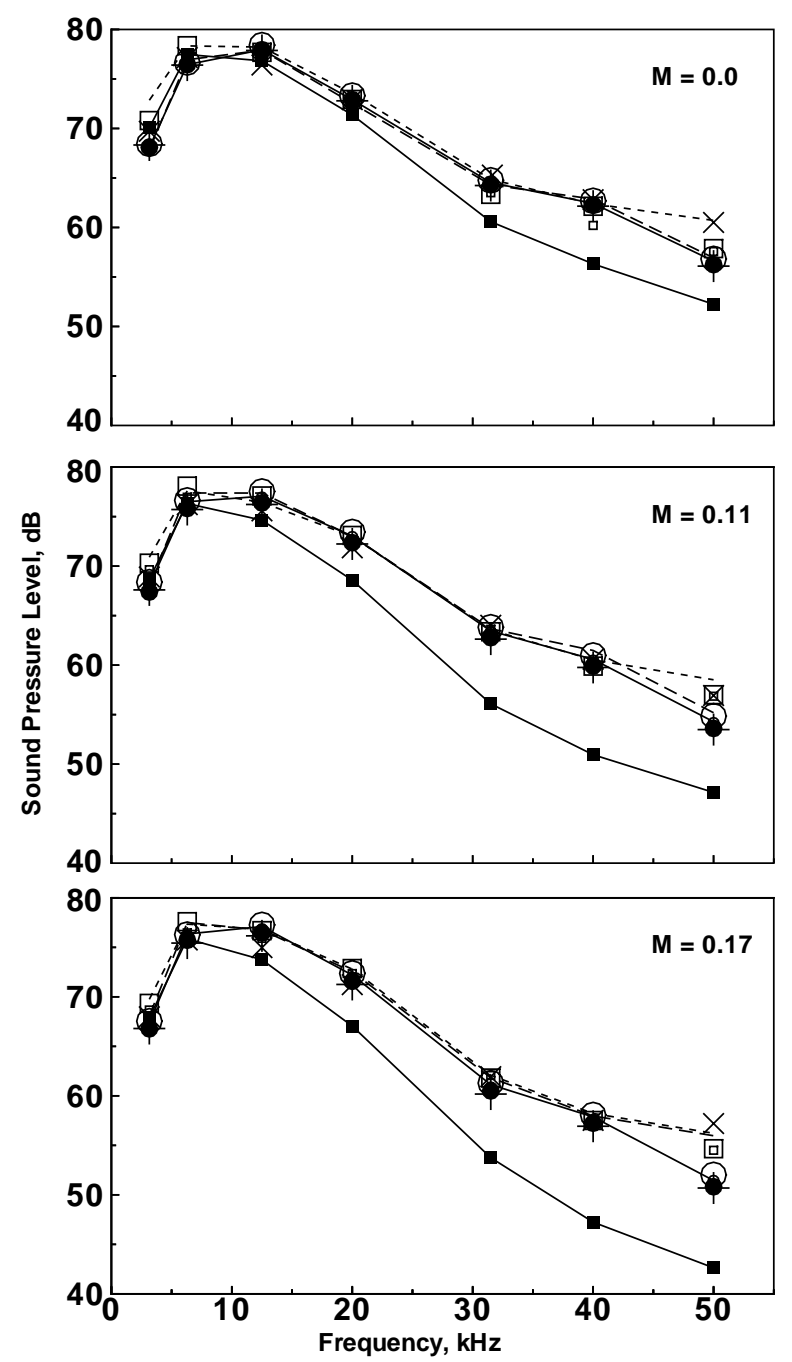

a) calibrator source for $M=0,0.11$, and 0.17 .

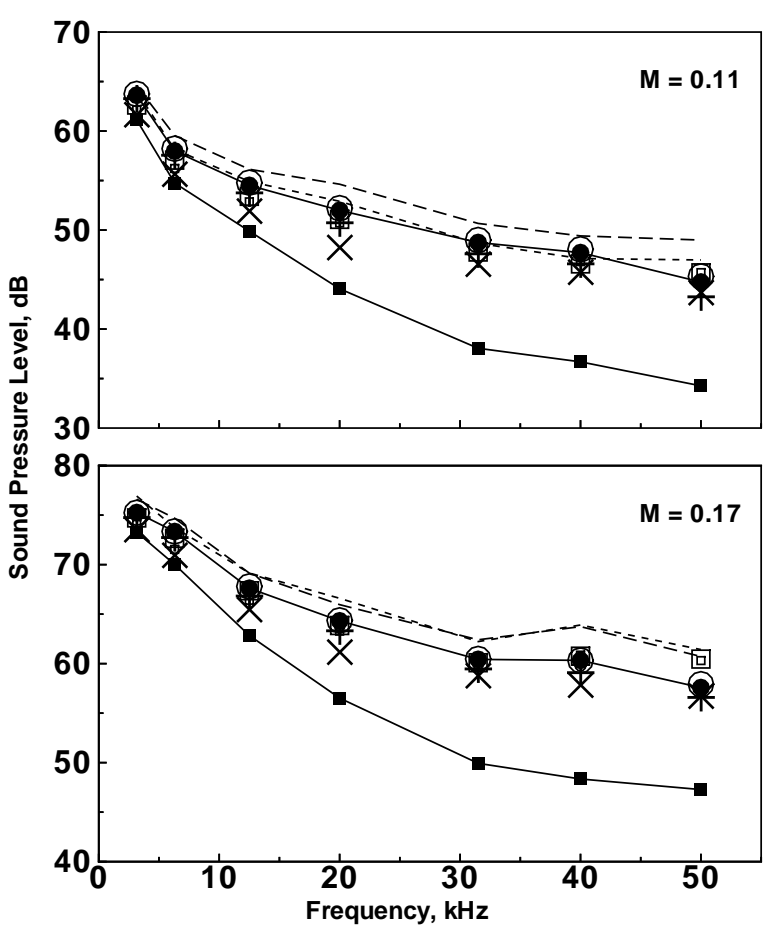

b) flat-edge flap for $M=0.11$ and 0.17 .

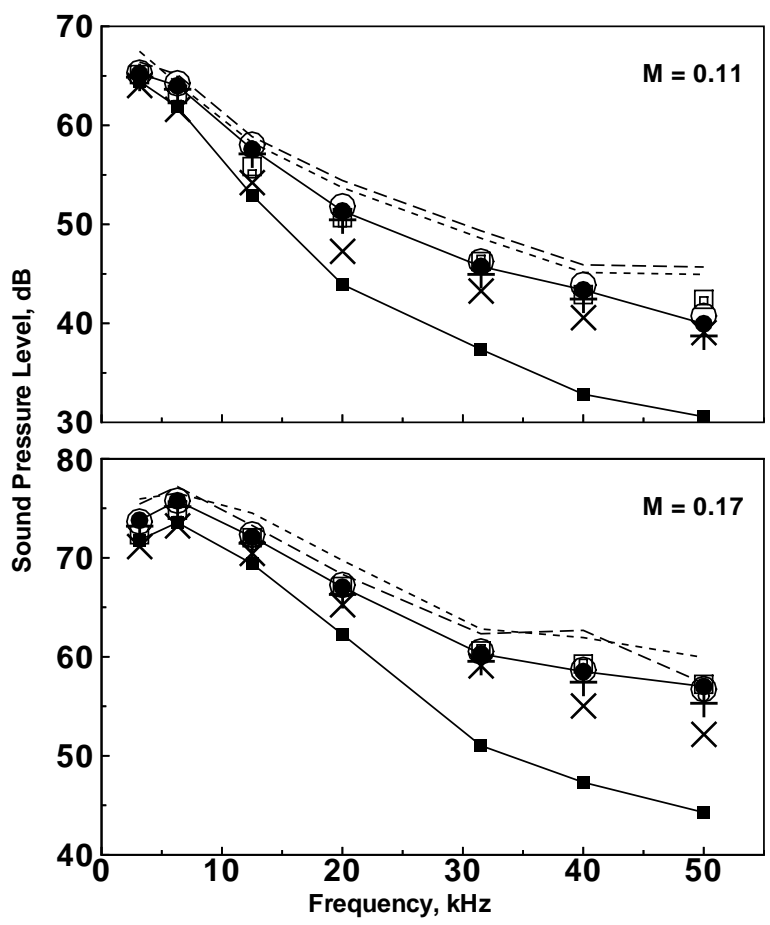

(c) contoured-edge flap for $M=0.11$ and 0.17 .

Figure 9. One-third Octave spectra using SADA, LADA, and single microphone from each. Integrated power levels are shown. 


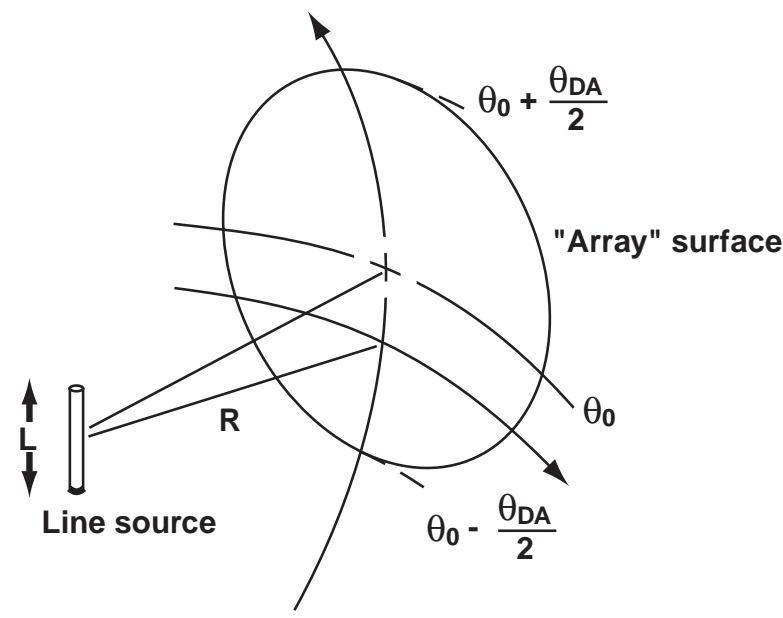

Figure 10. Sketch of an ideal line of length $L$ at the focal center of a spherical surface over which an "array" of solid angle $\theta_{D A}$ is defined.

analysis. In this regard, the main beamform lobe is only weakly dependent on the number of microphones in commonly designed arrays, such as those of this study.

"Measured" source distribution contours about a line source are shown in Fig. 11. The scanning plane was generated by offsetting the line source, from the center focus position of Fig. 10. The contour levels shown are $\Delta \mathrm{dB}_{L}$, defined as the output level of the array minus the total output of the source. The results are independent of $\mathrm{R}$, but depend on $L, \theta_{0}, \theta_{D A}$, and frequency. The $\theta_{0}\left(=39^{\circ}\right), \theta_{D A}$, frequency $(=40 \mathrm{kHz})$, and the contour presentation projections were chosen to generally correspond to the SADA and LADA projections of Fig. 7. The $L=2$ inches of length source is inclined to the projected plane by $39^{\circ}$ to simulate the flap-edge. The equivalent $L$ values for the tests were found to vary substantially as a function of frequency.

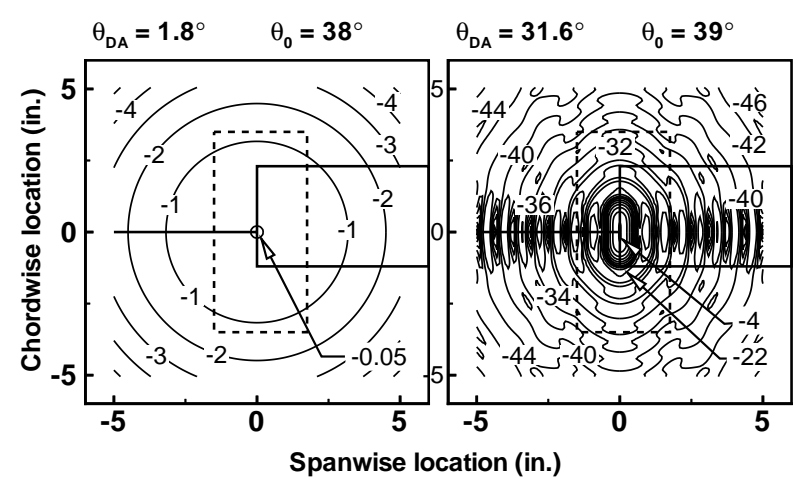

Figure 11. Source distribution contours as is theoretically measured for a 2 inch line source at $f_{1 / 3}=40 \mathrm{kHz}$. Projection is similar as the flap case of Fig. 7.
The choice of $L=2$ inches was arbitrary and was not made to match the appearance of Fig. 7. Also differing in appearance are the sidelobe details, which are specific only to this reference array and its processing, and thus can be ignored for the present purposes. Perhaps one thing that can be noted, by comparison of the Figs. 7 and 10, is that the actual source distributions are not lines but have dimensions in width as well as length.

Figure 12 shows plots of $\Delta d B_{L, \text { max }}$ versus a normalized frequency scale. $\Delta d B_{L, \text { max }}$ are the maximum levels of the contours which are found when the array is focussed at the center of the line. Curves are shown for an array orientation at $\theta_{0}=0^{\circ}$ and $39^{\circ}$. The normalized frequency is $k L \operatorname{Sin} \theta_{D A}$, where the wavenumber $k=2 \pi f / c_{0}$. With regard to results of Fig. 11, the $\Delta d B_{L, \text { max }}$ values of -.05 and $-3.8 \mathrm{~dB}$ for $\theta_{D A}=1.8^{\circ}$ and $31.6^{\circ}$, respectively, are found on the $39^{\circ}$ line at $k L \operatorname{Sin} \theta_{D A}=1.2$ and 19.6. The normalized frequency is a key result from the modeling. It says that measured array levels are reduced/increased, with respect to the total output of the line source, due to proportional increases/reductions in $f, L$, and/or $\theta_{D A}$. For sufficiently large $k L \operatorname{Sin} \theta_{D A}$, where the beamwidth of the main lobe is on the order of the length $L$ or smaller, $\Delta d B_{L \text {, max }}$ falls $3 \mathrm{~dB}$ per doubling of $k L \operatorname{Sin} \theta_{D A}$ for this incoherent source distribution, as one would expect.

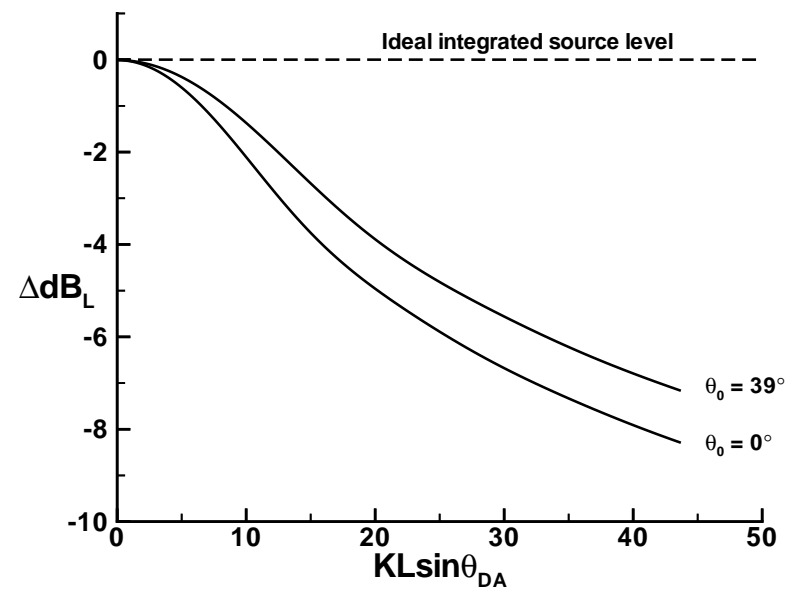

Figure 12. Peak output level of array minus the total output of a source of length $L, \Delta d B_{L \text {,max }}$, versus normalized frequency, for two $\theta_{0}$ array orientations.

The analytical model allows one to examine the effectiveness of integration procedures for different distributions of sources within integration region boxes. In Fig. 11, an integration area box is outlined on the contour. For an ideal integration procedure taken over 
the box (or any other box as long as it encloses the source), one should recover the total line source output, which is $0 \mathrm{~dB}$. In terms of Fig. 12, this corresponds to the $0-\mathrm{dB}$ line. Figure 13 is a presentation of error in integrated levels versus frequency for three cases. For each case, an integral procedure was carried out in a manner consistent with that of Eq. (5). Also carried out was one consistent with the simplified procedure described for Eq. (5), where a representative value $C_{l_{0}, n_{0}}$ is used, instead of $\mathrm{C}_{l, n}$. For these cases, $C_{l_{0}, n_{0}}$ was chosen for $l_{0}, n_{0}$ at the center of the integration box. This means the integration is referenced with respect to the integrated value of an ideal point source at the center of the box. The first comparison is that of a $L=3.5$ inches source at the center of a 7 by 3 -inch box. The full method of Eq. (5) gives integration errors of less than $+1 \mathrm{~dB}$. The simplified method for Eq. (5) gives more accurate results at lower frequencies. Both methods give little error at high frequency. When the $L=3.5$ inches source is displaced to 0.4 inches from the side of the box, the full method produces the least error of the two methods. (If $C_{l_{0}, n_{0}}$ were redefined to correspond to the offset position of the line source, it would have had less error than shown). The last case is where a large number of line sources fill the entirety of a 3.5 by 3.5 -inch box. Here the full method produces results with no error, whereas the simplified method has errors of about $-1 \mathrm{~dB}$ or more.

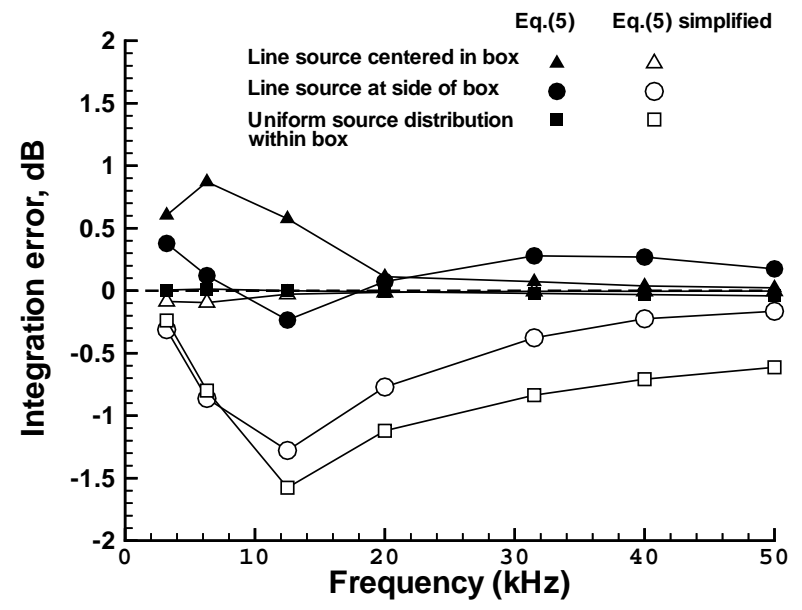

Figure 13. Integration errors for line and uniform sources in integration box.

Effect of Scattering by Free-Jet Shear Layer Turbulence. In the present study, the use of open jet testing allows the placement of the microphone arrays outside the flow (no microphone flow self noise) in an anechoic environment. However, as shown in Fig. 3, the sound produced by the model has to be transmitted through the turbulent shear layer at the open jet boundary on its way to the microphones. This causes the sound to be refracted, reflected, and scattered. The treatment for refraction and reflection is rather straightforward in principle. The mean refraction effects are accounted for in the beamform processing, as previously described. Reflection effects are generally very small, especially for low speed testing and nonsevere angles to the shear layer, as most all sound is transmitted through the shearlayer. ${ }^{28,29}$ Accounting for scattering of noise through the turbulence, however, is not as straightforward.

Figure 3 illustrates that sound is propagated along ray paths which are scattered with respect to the mean refracted ray path to a microphone. For a microphone, this spatially blurs the source. Also, due to Dopplertype effects in multiple turbulent encounters, energy is extracted from one frequency band and is redistributed in adjacent frequency bands. In the case of broadband noise spectral measurements by single microphones, the scatter effect can generally be neglected because this scatter may serve only to smooth the spectra without reducing level. However, for high frequency pure tones, scatter spreads the spectral width and reduces the peak amplitudes $28,30,31$. This occurs, it should be noted, with negligible loss in total spectral energy. The extent of the tone amplitude peak reduction has been found to depend on turbulent intensity ( $\propto$ Mach number $M$ ) and the ratio of the propagation path-length through the shear layer (generally taken as the shear layer thickness $\delta)$ to the acoustic wavelength $(\lambda)$.

Noise level reduction $\Delta d B_{S}$ due to scatter through free-jet shear layers, as found by Schlinker and Amiet ${ }^{28}$ (using a $50 \mathrm{~Hz}$ resolution bandwidth) and Ross ${ }^{31}$ (using a $125 \mathrm{~Hz}$ bandwidth) for tones are shown plotted versus the scatter parameter $M(\delta / \lambda)$ in Fig. 14. Because the bandwidth is larger for the Ross data, one would expect less level reduction; but this is not apparent. For these different tests, in-flow levels were monitored and corrections were made to prevent flow induced source changes from affecting the measured out-of-flow results. Figure 14, also, shows directional microphone (spherical mirror with a microphone at a focal point) system data for broadband noise rather than pure tones. The data were generated to calibrate one-third octave levels in an open-jet test for trailing edge noise. The levels shown were obtained for the present paper by subtracting spectra dB levels between Figs. 41 and 47 of Schlinker and Amiet ${ }^{32}$ and plotting versus approximated scatter parameter values. The 


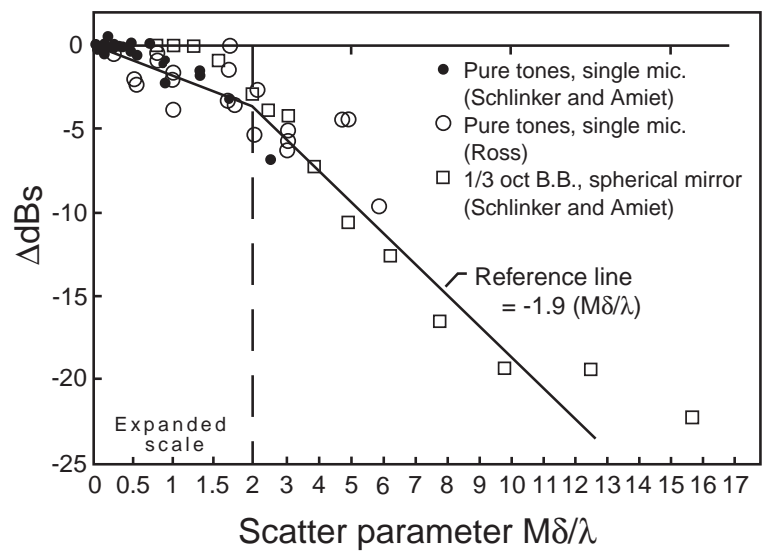

Figure 14. Measured level reductions due to shear layer turbulence scattering from Refs. (28), (31), and (32).

corresponding tunnel flow speeds for those figures were $M=0$ and $M=0.5$. The levels (that were subtracted) were the peak levels obtained from the mirror system while traversing in the streamwise direction past a broadband calibration source placed in the flow. The calculated scatter-parameter range shown is large for this data due to the higher flow speed compared to the $M=0.2$ to 0.3 speed ranges for the other data. None of the above authors chose to represent their data by any function, possibly due to the substantial data scatter. A reference line is given in Fig. 14 to represent the general trend (it is not a fit) of the data.

Figure 14 combines what may appear to be contradictory data. As mentioned, the reduced levels for pure tones are expected. But broadband one-third-octave levels are also reduced, which is contrary to what one may expect, at least if only a single microphone measurements were involved. However, these measurements are from a spherical mirror microphone system, which has a solid angle of $25^{\circ}$ (equivalent to $\theta_{D A}$ for the array) with respect to the source. In reconciling the data, one notes that a single microphone, or a location on the mirror surface, would "see" a direct radiated noise field summed with a specific scattered noise field. Here, the autospectral levels should not be reduced due to the scattering. Another single microphone or location in the surface would see similar autospectral levels. However, the cross-spectrum between locations would have levels less than the respective autospectra since they do not see identically scattered sound fields. The farther the locations are away from one another, the less coherent are the sound fields. In the limit, for a very large mirror surface (or array) and for a highly scattered noise field, the output levels of the system reduce greatly. This argument indicates that the precise levels shown (and thus the reference line levels) for the spherical mirror would actually depend on mirror size.

The scatter analysis above for the mirror system must also be applicable for the array. This is examined for the calibrator source data in Fig. 15 for the LADA sub-arrays of sizes $\theta_{D A}=9.9^{\circ}, 16.9^{\circ}, 25.5^{\circ}$, and $31.6^{\circ}$. The levels for $\Delta \mathrm{dB}_{\mathrm{S}}$ were approximated by

$$
\begin{aligned}
\Delta d B_{s} \approx & \left(d B_{\theta_{D A}}-d B_{\theta_{D A}}=2^{\circ}\right)_{M=0} \\
& -\left(d B_{\theta_{D A}}-d B_{\theta_{D A}}=2^{\circ}\right)_{M=.11, .17}
\end{aligned}
$$

where $d B_{\theta_{D A}}$ is the spectra level measured by a subarray of size $\theta_{D A}$ for the different Mach number cases. The first term should isolate the array/source size effect for the calibrator source. The second term has the combined effects of size and scatter. Their subtraction should isolate the level reduction due to the scatter effect. Any effect of change in source output level due to flow about the source should be eliminated in the subtraction. This all assumes that the actual source level is measured by the $\theta_{D A}=2.0^{\circ}$ sub-array. Also, it is noted that any significant change in directivity with speed could affect the comparisons. Directivity changes are observed to be mild to moderate in Fig. 9, so some concern is appropriate especially for high frequencies. The scatter parameters used for plotting were calculated using an average measured value for $\delta$ of 6.5 inches. In actuality, the effective $\delta$ varied from about 5.7 inches for the microphones at the bottom of the LADA to 8 inches for those at the top.

The results of Fig. 15 are generally bracketed by the reference line from Fig. 14. The two smaller and the two larger sub-arrays appear to scale well among themselves, except at high frequencies. As expected, the importance of this scatter phenomena is more severe for the larger arrays sizes. The substantial jump in importance of scatter for the two larger sub-arrays suggests that some threshold in array size may have been crossed where the coherence across the whole array face substantially drops. The reference line from Fig. 14 is mostly based on a $M=0.5$ speed condition for $\theta_{D A}=25^{\circ}$. In Fig. 14, the reference line for the low values of the scatter parameter shows a general overestimation of $\Delta d B_{S}$. This is in agreement with its comparison to the LADA data of Fig. 15. So, as the array size increases, $\Delta d B_{S}$ is larger and varies in general proportionally to the scatter scaling parameter. A heuristic modeling of this effect would be that a larger array effectively breaks-up into smaller sub-arrays (each with high local coherence) that are then summed by a weak"pressure-squared-summing" to give the reduced 
output. The effective sub-array sizes would decrease with increasing scatter parameter.

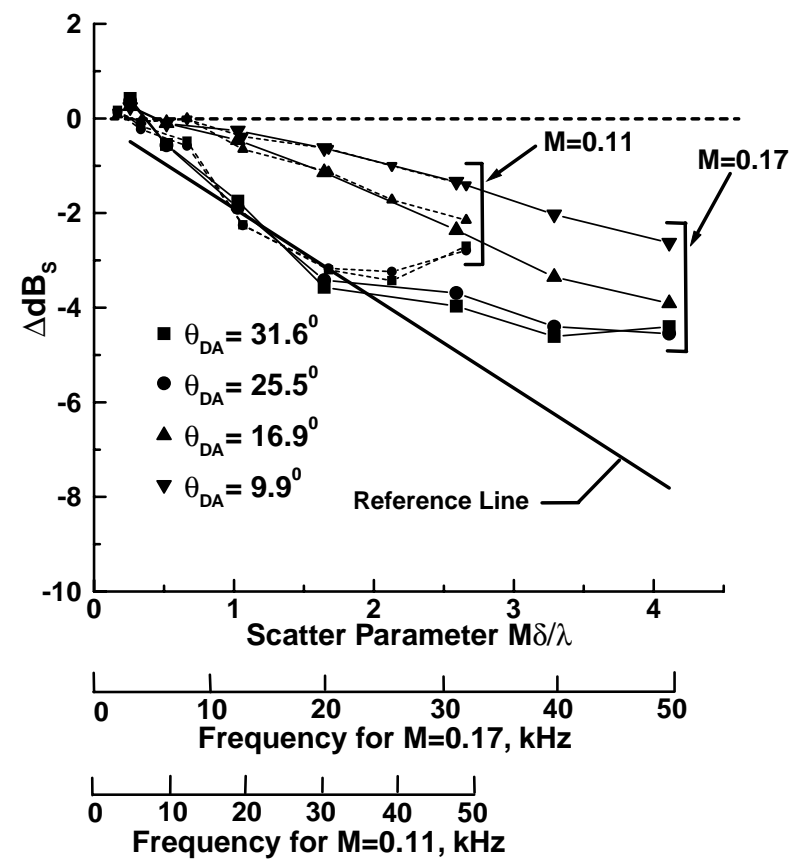

Figure 15. Measured level reductions due to scatter for the LADA. Reference line is from Fig. 14.

\section{Analysis of Results}

Taken together, the effects for source and array size, Fig. 12, and turbulence scatter, Fig. 15, appear large enough to explain the reduced levels for the LADA measurements compared to the SADA in Fig. 9. For either effect, there is nothing to indicate that by using large arrays, one will irretrievably lose portions of the noise. In fact, for the source-array size effect, integration approaches were shown to recover the total noise output of idealized noise distributions of uncorrelated sources.

Source Region Integration: The source region integration method of Eq. (5) is now examined with respect to recapturing the spectral energy of the array measurements. In Fig. 7, the source region contour maps for $40 \mathrm{kHz}$ have 3 by 7 -inch integration-region boxes defined for both the LADA and SADA. Although the source distributions varied with frequency, they generally stayed within the box. Note that for the SADA, the box truncates the main beampattern lobe. For the LADA, side lobes are present. These are permitted as long as the sources themselves are contained in the box. The integrated levels are listed in Fig. 7. For the SADA, it is seen that the integrated levels are only slightly higher than the maximum contour levels, as expected. However, the LADA inte- grated levels are substantially increased relative to the contour maximum levels and are close to that measured by the SADA. The success for the method for the full spectra is shown in Fig. 9. The integrated levels using Eq. (5) (represented by large closed symbols without lines) are seen to compare well with one another and with the blended SADA results. This shows success in recapturing the source spectra through integration. Also presented in Fig. 9 are integrated results for the simplified approach for Eq. (5) where $C_{l, n}$ is evaluated only at $l_{0}, m_{0}$ at the center of the integration region box. These results (smaller closed symbols) are good, being only being a little lower on the average. This agrees with the analysis for Fig. 13 for the idealized source integration.

The integrated levels agreed to within $1.5 \mathrm{~dB}$ for most data. This variation is quite reasonable considering possible source and model test variations, and calibration differences, between the SADA and LADA test efforts. Some error may be due to the summing of directivity variations over the face of the LADA, such as shown in Fig. 6. It was suggested that this effect may be small, but this was not examined in detail. Also, the integration method depends on the assumption of uncorrelated source distributions. The extent of source distribution correlation has not been established, but such effects on the present results are not apparent. Also, as part of the error analysis, the integration box size was varied from the present 3 by 7 -inch area with 0.25 -inch grid spacing to: 3 by 7 -inch with 0.125 -inch grid; 1.5 by 4 -inch with 0.125 -inch grid; and 12 by 12-inch with 0.25 -inch grid. For this analysis, the full Eq. (5) method was used. For the LADA point source data, all integrated spectral levels agreed within $0.5 \mathrm{~dB}$, except for the smallest box size where levels were uniformly down $0,-1$, and $-1.5 \mathrm{~dB}$ for $M=0,0.11$, and 0.17 , respectively. This appears to be quite consistent with the scatter effect extending beyond a "too-small" box.

The use of the diagonal-removal enhancement processing method of Eq. (4a) was examined and discussed with regard to Fig. 7. In Fig. 9, integrated spectral levels for this approach are given for the LADA and SADA. For clarity of presentation, the related measured spectra using this processing method are omitted. The integrated spectra (shown as crosses) were determined using Eqs. (5) and (5a) and the simplified $C_{l_{0}, n_{0}}$ method. The results for the calibrator source are seen to be fairly good and generally agree within 1 or $2 \mathrm{~dB}$ with the other integrated levels. For the flap edge noise cases, the levels are generally 2 to $5 \mathrm{~dB}$ lower for the LADA. The SADA results are generally less than $1 \mathrm{~dB}$ lower. The reason for the variability is not clearly 
known. It appears to depend on details of the source distribution because the degree of scatter effect should be the same for matched Mach numbers.

\section{DISCUSSION OF RESULTS AND IMPLICATIONS}

This study defines physical and processing phenomena involved in the measurement of noise sources by the use of arrays of different sizes. It shows that as long as basic criteria are met, one can retrieve total source levels by integration methods. This effort was made simpler by the fact that the noise studied is from relatively small but finite source regions, with intense levels compared to other sources. The anechoic environment of the arrays also enhanced the signal-to-noise.

Many applications of large arrays involve mounting microphones on surfaces (often the tunnel wall) in closed windtunnel test sections. The use of the diagonal-removal method is almost always used because of poor signal-to-noise conditions. Such measurement conditions are brought about not only from the effect of scatter, but also (and primarily) from turbulent boundary-layer wall-pressure fluctuations - which is a pseudo-noise contaminant—substantially removable by using this processing method. The present study offers only very limited support for the method. Although the integrated levels were generally good for the small calibrator source and for the SADA for all sources, the levels for the larger array were lower and more variable for the somewhat more spatially-extended flap sources. The present study dealt with this special processing method in only a limited way. More study of the ramifications of this method is highly recommended prior to extensive use of results from such processing for different sources in various tunnel environments.

The relative importance of the effect of scatter, in a closed tunnel turbulent boundary layer compared to the present free-jet shear layer, is not known. However, if the previously discussed results of Storms, et al. ${ }^{22}$ are any indication, the scatter effect may be very important, especially being combined with the use of the diagonalremoval processing discussed above. (It should be noted that the $6 \mathrm{~dB}$ drop experienced by Storms, et al. in integrated levels at about $13 \mathrm{kHz}$ could, in addition, be related to the source size effect discussed below.) The whole issue of scatter is not straightforward and is intermingled with the issue of array size. For example, for larger arrays, scatter undoubtedly contributes to the problem of separating out multiple sources. For smaller arrays, scatter is less important but the issue of separating multiple sources may not arise because the array's resolution may not be sufficient to identify multiple sources. In any event, for large arrays, it is recommended that specific calibrations to determine scatter effect amplitude be performed for different test setups. Also, it is advisable to enhance the crossspectral matrices of data sets by subtracting corresponding background data to substantially account for the basic test stand and tunnel environment noise, as well as microphone pseudo-noise. This may eliminate the need to use the possibly troublesome diagonalremoval method. Given good signal-to-noise conditions, larger arrays can be used to separate sources as long as infringing side lobes are accounted for. In this regard, processing different sub-array sizes can be used diagnostically to move sidelobes to avoid overlap between sources. When using large arrays, different sub-array groupings could help validate that the character of the source being studied is properly defined by the full array. Also, cross-spectra between the subarrays may reveal much about the source.

A high noise floor (poor dynamic range) can limit the usefulness of an array, both qualitatively and quantitatively. Of particular interest here is accounting for extended sources in the presence of compact sources. To show the difficulty, an example is now given for a test configuration of a previous study ${ }^{33,2}$. In this study, a system of microphones was used, but directional array techniques were not. The noise studied was from an 18-inch span airfoil with a rounded tip. The tip noise was emitted from a turbulent tip-vortex rollup region of 0.5 -inch spanwise extent at the trailing edge (TE). The level of this tip noise was measured (by correlation techniques) to be very nearly equal to the TE noise from the airfoil over the remaining 17.5 inch span, in the frequency range from 5 to $15 \mathrm{kHz}$. Consider what would be measured, according to the array level response determined from Fig. 12 (this would be exclusive of any scatter effects), when using the SADA and LADA arrays at 10 and $15 \mathrm{kHz}$. Table 1 lists the relative $d B$ levels that would appear on resulting contour plots of the array output over the source region. A contour plot for the LADA at $10 \mathrm{kHz}$ would render a level at the tip of $\mathrm{dB}_{0}-.2$, where $\mathrm{dB}_{0}$ is the actual tip noise. The level at the TE region away from the influence of the high tip noise levels would be $\mathrm{dB}_{0}-8.8$, where $\mathrm{dB}_{0}$ is the total TE noise level (being equal to that of the tip). Here, signal-to-noise is a major issueit would appear to make the presence of the TE noise (one-half of the total noise) almost invisible to the large array, when effects such as scatter are added in. The situation is seen to be worse by $2 \mathrm{~dB}$ at $15 \mathrm{kHz}$. The results for the SADA are seen to be less severe at $\mathrm{dB}_{0}-2.2$. This $-2.2 \mathrm{~dB}$ is constant at different frequencies because of the constant resolution processing of SADA. It should be noted that, it is the authors' understanding that it is not unusual for 
Table 1. Output of arrays, $\Delta \mathrm{dB}_{\mathrm{L}, \mathrm{max}}$ based on Fig. 12, in measuring extended line sources of Ref. 33.

\begin{tabular}{|c|c|c|c|c|}
\hline & \multicolumn{2}{|c|}{ Output of LADA } & \multicolumn{2}{|c|}{ Output of SADA } \\
\hline & $f=10 \mathrm{kHz}$ & $f=15 \mathrm{kHz}$ & $f=10 \mathrm{kHz}$ & $f=15 \mathrm{kHz}$ \\
\hline Tip Noise, $\mathrm{L}=.05$ in & $-.2 \mathrm{~dB}$ & $-.4 \mathrm{~dB}$ & $0 \mathrm{~dB}$ & $0 \mathrm{~dB}$ \\
\hline TE Noise, $L=17.5$ in. & $-8.8 \mathrm{~dB}$ & $-10.6 \mathrm{~dB}$ & $-2.2 \mathrm{~dB}$ & $-2.2 \mathrm{~dB}$ \\
\hline
\end{tabular}

contributions of levels below $-6 \mathrm{~dB}$ to be systematically excluded in the application of some integration algorithms.

An additional point is made with regard to difficulties in measuring extended sources. The smaller SADA array has been used to measure slat noise using the present test setup in the QFF (no data are presented in this paper). It has been found that the presence of extraneous noise by small irregularities (which can be a small contribution to the total noise, but which is concentrated spatially) can interfere with the distributed slat noise measurements. In these cases, the contour maps appear to have the slat noise partially submerged over some regions. One must account for the fact that the true importance of the ( $L=36$ inch) slat is suppressed by some $4.6 \mathrm{~dB}$. However, proper integration of the whole region should account for the true relative importance.

\section{CONCLUSIONS}

This study defines physical and processing phenomena involved in the measurement of aeroacoustic sources by the use of microphone arrays of different sizes. Two arrays and sub-arrays of different sizes, ranging from $2^{\circ}$ to $31.6^{\circ}$ of solid angle with respect to the source region, were tested outside the flow in an anechoic open-jet flow facility. The noise sources measured were several flap-edge configurations and a calibrator source. The following conclusions can be drawn:

A small array, such as the SADA, in an anechoic environment can be designed to measure directivity and spectra of selected portions of a noise component. A small size permits easy movement about a model under study and the face of the array can be confined to a small portion of the directivity (to prevent directivity smearing). Processing can be tailored to keep the measurement resolution constant with frequency so that the output spectra do not require correction. A small array cannot, however, resolve and separate the details of source distributions.
A large array, such as the LADA, can be designed to locate and separate noise source details over selective regions of a noise component. It is not generally suitable for directivity measurements. Also, with the finer resolution, the spectra are shown for large arrays to measure substantially decreased levels compared to smaller arrays. Analyses were performed to understand and account for the factors affecting measurements.

- First, an analytical model, for an ideal array and line source, was used to find that levels measured by an array are reduced (compared to a source's total output) directly with increases in array size, source size, and frequency. Second, the effect of noise level reduction due to scattering by shear layer turbulence was examined using the present data and those of previous studies. Taken together, the two effects were sufficient to explain spectral level differences between the arrays of different sizes.

- An important result of the paper is that integration techniques, which were used to sum the noise measured over survey regions for the two arrays, were able to recover the total noise spectral output of the different sources. Such a demonstration is necessary, in order to establish confidence in the array method. The use of special purpose diagonalremoval processing was found to have variable success in determining accurate integrated results. The results suggest the influence of source distribution on integrated levels for large arrays. More examination of the method is required.

Implications from the present open-windtunnel study are discussed with regard to testing in closed testsection tunnels. For additional types of problems existing for closed tunnels, processing strategies are proposed to assist quantitative interpretations. For both open and closed tunnels, a problem of concern is to properly account for distributed noise sources. In particular, large arrays naturally suppress the measured levels of extended sources in comparison to more compact sources. Integration techniques can be used to recover the contributions of extended-sources to the 
total noise; but unless care is taken, one may ignore or systematically discard this contribution, such as when trying to deal with levels of noise floor contamination.

\section{$\underline{\text { ACKNOWLEDGMENTS }}$}

The authors wish to thank our colleagues Drs. K.R. Meadows and W.W. Hunter for their essential and longlasting contributions to the project. We also acknowledge Daniel J. Stead and Tim L. Lavallee, of LockheedMartin Engineering and Sciences Co., for data processing and software development support. The authors also gratefully acknowledge Ron Verhapen of Wyle Laboratories for data acquisition system support.

\section{REFERENCES}

1. Brooks, T.F. and Hodgson, T.H., "Trailing Edge Noise Prediction from Measured Surface Pressures," J. Sound \& Vibration, Vol. 78, No. 1, Sep. 1981, pp. 69-117.

2. Brooks, T.F., Pope, D.S., Marcolini, M.A., "Airfoil SelfNoise and Prediction," NASA Reference Publication 1218, July, 1989.

3. Kendall, J.M., Jr., "Airframe Noise Measurements by Acoustic Imaging," AIAA Paper 77-55, AIAA $15^{\text {th }}$ Aerospace Sciences Meeting, Los Angeles, CA, January, 1977.

4. Grosche, F.R., Stiewitt, H., and Binder, B., "Acoustic Wind-Tunnel Measurements with a Highly Directional Microphone," AIAA Journal, Volume 15, Number 11, pp. 1590-1596, 1977.

5. Schlinker, R.H., "Airfoil Trailing Edge Noise Measurements With a Directional Microphone," AIAA Paper 77-1269, $4^{\text {th }}$ AIAA Aeroacoustics Conference, Atlanta, GA, October, 1977.

6. Grosche, F.R., Schneider, G., and Stiewitt, H., "Wind Tunnel Experiments on Airframe Noise Sources of Transport Aircraft," AIAA Paper 97-1642, $3^{\text {rd }}$ AIAA/CEAS Aeroacoustics Conference, Atlanta, GA, May, 1997.

7. Bent, P.H., Horne, W.C., and Watts, M.E., "Airframe Noise Scaling and Source Localization", Noise-Con 96 Proceedings. pp. 145-150, Seattle, WA, Sep. 29-Oct. 2, 1996.

8. Soderman, P.T., and Noble, S.C., "Directional Microphone Array for Acoustic Studies of Wind Tunnel Models," Journal of Aircraft, pp. 169-173, 1975.

9. Billingsley, J., and Kinns, R., "The Acoustic Telescope," Journal of Sound and Vibration, Vol. 48, No. 4, pp. 485$510,1976$.
10. Brooks, T.F., Marcolini, M.A., and Pope, D.S., "A Directional Array Approach for the Measurement of Rotor Noise Source Distributions with Controlled Spatial Resolution," Journal of Sound and Vibration, Vol. 112, No. 1, pp. 192-197, 1987.

11. Marcolini, M.A., and Brooks, T.F., "Rotor Noise Measurement Using a Directional Microphone Array," Journal of the American Helicopter Society, pp. 11-22, 1992.

12. Blacodon, D., Caplot, M., and Elias, G., "A Source Localization Technique for Helicopter Rotor Noise," $11^{\text {th }}$ AIAA Aeroacoustics Conference, Sunnyvale, CA, Oct. 1987.

13. Elias, G., "Noise Source Localization with Focussed Antenna for Reduction Purposes," Presented at the Science et Defence Conference, France, pp. 1-13, May 1990 (Transl. into ENGLISH from Science et Defence, France)

14. Gramann, R.A. and Mocio, J.W., "Aeroacoustic Measurements in Wind Tunnels using Adaptive Beamforming Methods," Journal of the Acoustical Society of America, Vol. 97, No. 6, pp. 694-701, June 1995.

15. Michel, U., Barsikow, B., Helbig, J., Hellmig, M., and Schuttpelz, M., "Flyover Noise Measurements on Landing Aircraft with a Microphone Array," AIAA Paper 98-2336, $4^{\text {th }}$ AIAA/CEAS Aeroacoustics Conference, Toulouse, France, June 2-4, 1998.

16. Watts, M.E., Mosher, M., and Barnes, M.J., "The Microphone Array Phased Processing System (MAPPS)," AIAA Paper 96-1714, $2^{\text {nd }}$ AIAA/CEAS Aeroacoustics Conference, State College, PA, May, 1996.

17. Humphreys, W.M., Brooks, T.F., Hunter, W.W., and Meadows, K.R., "Design and Use of Microphone Directional Arrays for Aeroacoustic Measurements," AIAA Paper 98-0471, 36 ${ }^{\text {st }}$ Aerospace Sciences Meeting \& Exhibit, Reno NV, Jan. 12-15, 1998.

18. Underbrink, J.R., "Practical Considerations in Focused Array Design for Passive Broadband Source Mapping Applications," Master's Thesis, The Pennsylvania State University, May, 1995.

19. Dougherty, R.P. and Stoker, R.W., "Sidelobe Suppression for Phased Array Aeroacoustic Measurements," AIAA Paper 98-2242, $4^{\text {th }}$ AIAA/CEAS Aeroacoustics Conference, Toulouse, France, June 2-4, 1998.

20. Underbrink, J.R. and Dougherty, R.P., "Array Design for Non-Intrusive Measurement of Noise Sources," Noise- 
Con 96 Proceedings. pp. 757-762, Seattle, WA, Sep. 29-Oct. 2, 1996

21. Johnson, D.H., and Dudgeon, D.E., Array Signal Processing, Prentice Hall, 1993.

22. Storms, B.L., Ross, J.C., Horne, W.C., Hayes, J.A., Dougherty, R.P., Underbrink, J.R., Scharpf, D.F., and Moriarty, P.J., "An Aeroacoustic Study of an Unswept Wing with a Three-Dimensional High-Lift System," NASA TM-1998-112222, February 1998 (Publicly available Feb. 2000)

23. Herkes, W.H. and Stoker, W.H., "Wind Tunnel Measurements of the Airframe Noise of a High-Speed Civil Transport," AIAA Paper 98-16338, 36 ${ }^{\text {th }}$ Aerospace Sciences Meeting \& Exhibit, Reno, NV, Jan. 12-15, 1998.

24. Mosher, M., "Phased Arrays for Aeroacoustic Testing: Theoretical Development," AIAA Paper 96-1713, $2^{\text {nd }}$ AIAA/CEAS Aeroacoustics Conference, State College, PA, May 6-8, 1996.

25. Pierce, A.D., Acoustics: An Introduction to Its Physical Principles and Applications, McGraw-Hill Book Company, 1981.

26. Mosher, M., Watts, M.E., Jaeger, S.M., and Jovic, S., "Calibration of Microphone Arrays for Phased Array Processing," AIAA Paper 97-1678, $3^{\text {rd }}$ AIAA/CEAS Aeroacoustics Conference, Atlanta, GA, May 12-14, 1997.
27. Meadows, K.R., Brooks, T.F., Humphreys, W.M., Hunter, W.W., and Gerhold, C.H., "Aeroacoustic Measurements of a Wing-Flap Configuration," AIAA Paper 97-1595, $3^{\text {rd }}$ AIAA/CEAS Aeroacoustics Conference, Atlanta, GA, May 12-14, 1997.

28. Schlinker, R.H., and Amiet, R.K., "Shear Layer Refraction and Scattering of Sound", AIAA Paper 80973, 1980 (also NASA CR 3371, Dec. 1980).

29. Amiet, R.K., "Refraction of Sound by a Shear Layer," Journal of Sound and Vibration, Vol. 58, No. 3, pp. 467482, 1978.

30. Ahuja, K.K., Tanna, H.K., and Tester, B.J., "An Experimental Study of Transmission, Reflection and Scattering of Sound in a Free Jet Flight Simulation Facility and Comparison with Theory," Journal of Sound and Vibration, Vol. 75, No. 1, pp. 51-85, 1981.

31. Ross, R., "Spectral Broadening Effects in Open Wind Tunnels in Relation to Noise Assessment," AIAA Journal, Vol. 19, No. 5, pp. 567-572, May 1981.

32. Schlinker, R.H., and Amiet, R.K., "Helicopter Rotor Trailing Edge Noise," NASA Contractor Report 3470, Nov. 1981.

33. Brooks, T.F. and Marcolini, M.A., "Airfoil Tip Vortex Formation Noise," AIAA Journal., Vol. 24, No. 2, pp. 246-252, Feb. 1986. 